\title{
Assessment of muscle activity using electrical stimulation and mechanomyography: a systematic review
}

\author{
Raphael Uwamahoro ${ }^{1,2}$, Kenneth Sundaraj ${ }^{j^{*}}$ and Indra Devi Subramaniam ${ }^{3}$
}

\author{
${ }^{*}$ Correspondence: \\ kenneth@utem.edu.my \\ ${ }^{1}$ Fakulti Kejuruteraan \\ Elektronik \& Kejuruteraan \\ Komputer, Universiti Teknikal \\ Malaysia Melaka, Tunggal, \\ Malaysia \\ Full list of author information \\ is available at the end of the \\ article
}

\begin{abstract}
This research has proved that mechanomyographic (MMG) signals can be used for evaluating muscle performance. Stimulation of the lost physiological functions of a muscle using an electrical signal has been determined crucial in clinical and experimental settings in which voluntary contraction fails in stimulating specific muscles. Previous studies have already indicated that characterizing contractile properties of muscles using MMG through neuromuscular electrical stimulation (NMES) showed excellent reliability. Thus, this review highlights the use of MMG signals on evaluating skeletal muscles under electrical stimulation. In total, 336 original articles were identified from the Scopus and SpringerLink electronic databases using search keywords for studies published between 2000 and 2020, and their eligibility for inclusion in this review has been screened using various inclusion criteria. After screening, 62 studies remained for analysis, with two additional articles from the bibliography, were categorized into the following: (1) fatigue, (2) torque, (3) force, (4) stiffness, (5) electrode development, (6) reliability of MMG and NMES approaches, and (7) validation of these techniques in clinical monitoring. This review has found that MMG through NMES provides feature factors for muscle activity assessment, highlighting standardized electromyostimulation and MMG parameters from different experimental protocols. Despite the evidence of mathematical computations in quantifying MMG along with NMES, the requirement of the processing speed, and fluctuation of MMG signals influence the technique to be prone to errors. Interestingly, although this review does not focus on machine learning, there are only few studies that have adopted it as an alternative to statistical analysis in the assessment of muscle fatigue, torque, and force. The results confirm the need for further investigation on the use of sophisticated computations of features of MMG signals from electrically stimulated muscles in muscle function assessment and assistive technology such as prosthetics control.
\end{abstract}

Keywords: Muscle activity, Muscle mechanics, Muscle assessment, Mechanomyography, Electrical stimulation included in the article's Creative Commons licence and your intended use is not permitted by statutory regulation or exceeds the permitted use, you will need to obtain permission directly from the copyright holder. To view a copy of this licence, visit http://creativecommons.org/ licenses/by/4.0/. The Creative Commons Public Domain Dedication waiver (http://creativecommons.org/publicdomain/zero/1.0/) applies to the data made available in this article, unless otherwise stated in a credit line to the data. 


\section{Introduction}

The musculoskeletal system has been determined to play an extensive role in the locomotor function of the body. Excited by the microstimulation of the central nervous system, the system acts on the skeletal joints, generating the force required to provoke dynamic motion and posture. This force provides the joint actuator with the integral summation needed for individual muscle activation [1]. To date, the electrical response from muscle contraction has been recorded using electromyography (EMG) [2], which is detected through electrodes placed on the surface of the muscle of interest. This signal is produced by the overall contribution of active muscles beneath the skin. Thus, the recorded EMG could be contaminated by active neighboring muscles. This contamination, which is often referred to as crosstalk, has been reported in muscle coordination, motion analysis, muscle task evaluation, and prosthesis control. Different muscles are synchronously activated to contribute to a single task. Thus, scientists were aware of proper methods of screening the function of unique muscle with reduced disturbances from neighboring muscles.

NMES has been identified as an alternative tool to voluntary activation in order to generate muscle contraction; it has been determined to be an important feature to selectively activate desired muscles. Nevertheless, the changes in EMG patterns over time [3, 4] and electrical interference [5] are considered deterministic factors that might affect its proper interpretations when the targeted muscle is subjected to electrical quantities. Consequently, the development of piezoelectric, microphones, and accelerometers showed appropriate detection of the mechanical signals from the surface of skeletal muscles at a low frequency, which is known as MMG signal [6] not contaminated by electrical noise. Indeed, MMG signal represents the mechanical manifestation of muscle activity [7] and further indicates the neurophysiology reflected by the mechanical counterpart to the electrical activity of unfused active motor units [5]. Attempts made in the assessment of crosstalk [8], quantification [9], and applications in assistive technology [10] support MMG signal as an alternative to EMG signal for the screening of muscle function [11], in terms of fatigue [12], muscle force [13], and its derivative (torque) [14] as well as for prosthesis control [15] and the detection of myopathies [16].

Indeed, the frequency component of MMG signals can provide essential information on the contraction features of the muscles of interest coupled with information on the muscle fiber types and composition. For example, electrical activation of the slow- and fast-twitch muscle fibers in soleus (SOL) and vastus lateralis (VL) showed lower MMG-median frequency in SOL than in VL [17]. The mean frequency (MF) of EMG signals has been determined to mirror the conduction velocity of recruited motor units (MUs). The firing rate of recruited MUs range in 10 and $40 \mathrm{~Hz}$ EMG spectrum [18], whereas MMG appears at lower $10 \mathrm{~Hz}$ [19]. EMG is often influenced by the shape of the action potential, whereas the duration of elementary MMG and the action potential converge. Using isometric muscle action, the MMG-EMG crossspectrum showed that unlike MMG, EMG has a low sensitivity for different types of MUs and composition [18] with long-lasting (100 $\mu \mathrm{s})$ MU actuators than MUAP $(10 \mu \mathrm{s})$ [19]. Thus, inference behind the features of MMG is crucial for muscle activity assessment. This fact supports the combination of MMG and NMES to provoke and record the muscle contraction, respectively. As a passive form of muscle 
recovery, NMES, which aims to activate and improve the function of peripheral muscles through electrical intensity delivered to intact motor nerves, found use in treating individuals suffering from dyspnea or muscle weakness [20]. Hence, the muscle integrity and force generation can be further improved by NMES-induced training, which can lead to increased muscle size and fiber type transformation [21]. In particular, MMG obtained after NMES is crucial in monitoring the reduction in muscle activation during the development of fatigue [22].

Early studies have highlighted the validity and reliability of field electrode stimulation and MMG in terms of analyzing skeletal muscle function. The available techniques for assessing human skeletal muscle activity during a diverse range of applications should be comprehensively reviewed. Few review articles have identified the muscle weakness in geriatric [20], gait speed in the lower limb [23], and the degree of spinal cord injury (SCI) [24], but the use of MMG from electrical excitation of skeletal muscles has been limited to theoretical concepts [21, 22]. Hence, this review assesses published studies on the use of MMG through NMES quantities for the assessment of muscle function. Specifically, this review identifies electrically induced MMG parameters related to health conditions of the upper limb, lower limb, and other areas of the body, and further analyzes the results from different experimental protocols used for specific muscles. This study also clarifies typical measures associated with the strengths and weaknesses of MMG from NMES-based recording methods for muscle performance. Then, assessment techniques of the muscle functions are discussed, suggesting exploratory techniques to provide novel insights that could lead to further improvements.

Collectively, intriguing issues have been concisely analyzed based on their applications to further explore the relationship between the physiological mechanism underlying a muscle and the level of physical function. In particular, this review identified studies that examined fatigue and endurance during exercise and stretching, force, power, torque, and muscle stiffness. In many respects, several studies reported the changes in MMG parameters caused by sensor reposition. Thus, motivated other research to develop and validate NMES electrodes and MMG sensors, as detailed in this review.

Advancements have also been made to discover the origin and characteristics of MMG, further discussing four terminologies: phonomyography (PMG), vibromyography (VMG), kinemyography (KMG), and acceleromyography. Their temporal and spectral feature representations have been used in evaluating muscle fatigue, force, and torque and in clinical monitoring using polynomial regression and linear correlation analysis. These provide evidence of MMG and NMES for the assessment of skeletal muscle function to synthesize the insights for stimulating and recording approaches in terms of the protocol design, equipment maneuvers, and performance evaluation in clinical and experimental settings. Although the review did not relate to machine learning, few studies have used support vector regression (SVR), support vector machine (SVM), and artificial neural network (ANN) to enhance the reliability of electrically evoked MMG characteristics in force, torque, and fatigue assessment. It is likely that these findings are deemed useful to further understand the muscle function and restoration and could guide for further action when specific features of MMG signals through NMES programs are required for a specific experiment. 


\section{Methods}

\section{Data extraction}

The studies analyzed on this research have been extracted from Scopus and SpringerLink electronic databases. These studies were identified by taking into account research works published between 2000 and 2020 using a combination of search keywords as shown in Fig. 1. This search yielded a significant number of studies that were then screened using inclusion and exclusion criteria. Initial selection evaluated the titles to identify studies on MMG through electrical muscle stimulation before full-text screening. The data extraction technique removed unreliable and nonpeer-reviewed articles and any duplicates. Studies with unclear methodology and articles written in language rather than English were removed from this review. In total, 336 articles, which were identified using this search strategy, were individually examined for eligibility for inclusion in the final

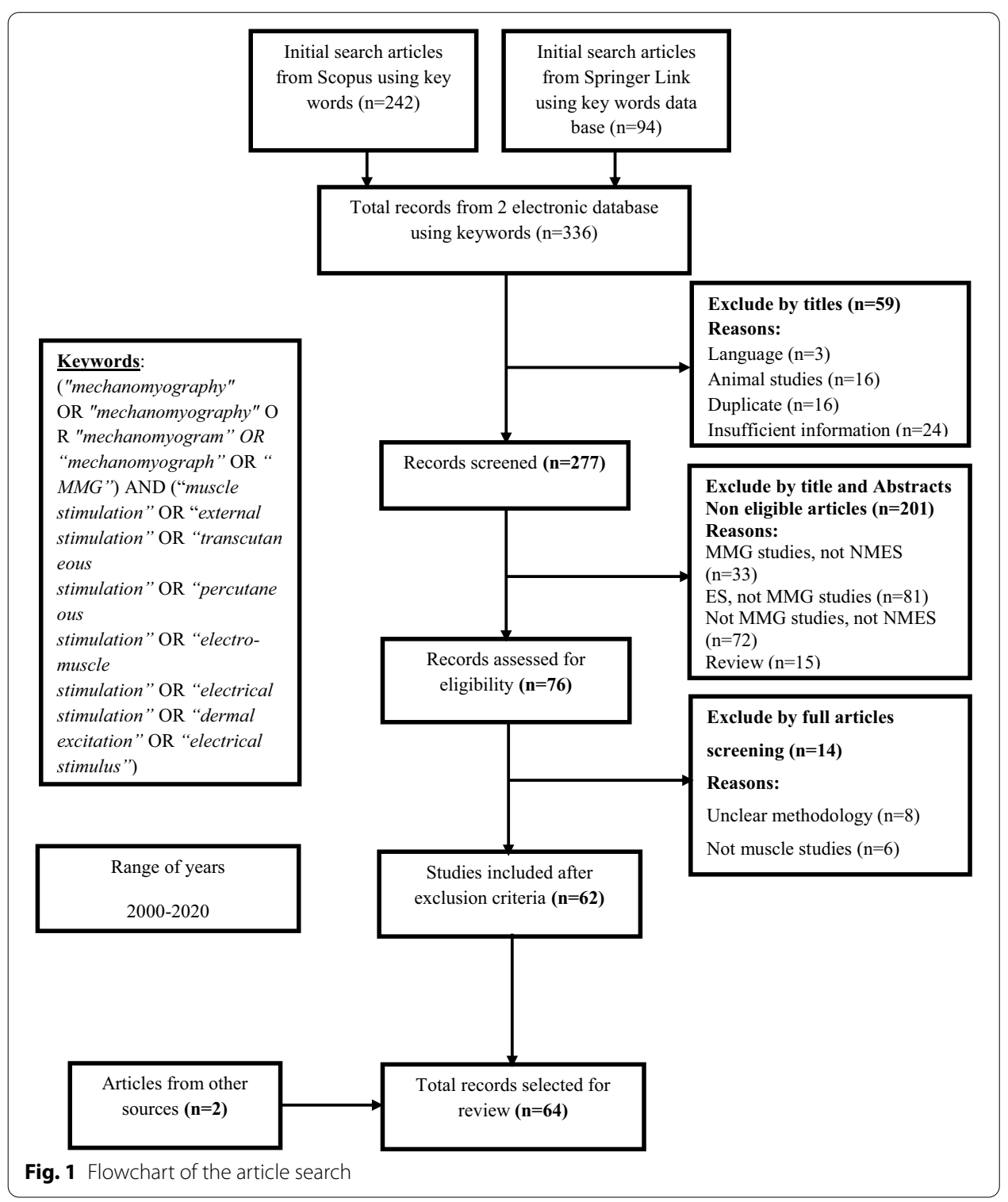


study. However, 3 of the 336 articles were not included in the final screening as the manuscripts were not written in English language; in addition, 16, 16, and 24 other articles were removed from the final set as they discussed animal studies, were duplicate articles, and did not provide sufficient information. Moreover, 201 additional articles were eliminated after a review of their titles and abstracts was conducted, and this review further indicated that 33 of the studies evaluated MMG but did not include NMES, 81 discussed NMES but did not perform an MMG analysis, 72 did not evaluate MMG nor electromyostimulation, and 15 were review articles. An additional screening resulted in the exclusion of 8 studies due to an unclear methodology, and 6 other studies were further removed because they did not evaluate the muscles. The final screening resulted into 62 articles that were considered for the review, as shown in Fig. 1. Using the same eligibility criteria, screening of the bibliography in the 62 studies identified 2 articles that provided supporting information to avoid ambiguity not clarified in all the 62 studies.

\section{Data analysis}

In total, 64 articles related to NMES and MMG were considered in this review, of which 18 highlight the application of electrically elicited MMG in fatigue assessment, 6 argued stiffness, 9 evaluated for muscle contributions to joint torque, and 4 studies addressed the assessment of force. Moreover, this investigation yielded six studies discussing the further validation of NMES technique for specific substrates in MMG signal acquisition; meanwhile, one study adopted respiratory muscle activity from the electrically elicited motor nerve. Furthermore, 13 and 6 articles, involving electrical activation of muscles, have examined the reliability of MMG parameters under experimental and clinical settings, respectively, whereas one article evaluated an approach involving MMG and electrically triggered ultrafast imaging system in real time to record small fiber contraction in the region of interest of a target muscle. NMES parameters, MMG features, type of sensors used, electrode sites, and the experimental protocol were then extracted from each article.

\section{Analysis and discussion of MMG and NMES Reliability of MMG measurements Sensor sites}

As shown in Table 1, four records [25-28] have been identified to examine the effects of electrode sites on MMG. The authors of one of these studies [25] found that the skinfold thickness can influence the EMG M-waves and MMG gross lateral movement recorded from electrically excited VL and rectus femoris (RF) muscles. As a measurement of the muscle belly displacement, the findings obtained in another previous study [26] revealed the efficacy of tensiomyography (TMG) in terms of detecting muscle contractile parameters with varying inter-electrode distance (IED). However, due to electrodes repositioning, a decrease in IED from \pm 5 to $\pm 3 \mathrm{~cm}$ has been observed to lower the maximum muscle displacement. Thus, these researchers claimed that IED should be maintained during experiments that require electrode repositioning.

In clinical settings, where muscle disuse might lead to physiological weakness, TMG can be used for estimating muscle mass recovery. Using ultrasound scans of the vastus medialis obliquus (VMO) and vastus medialis longus (VML), the thickness and 


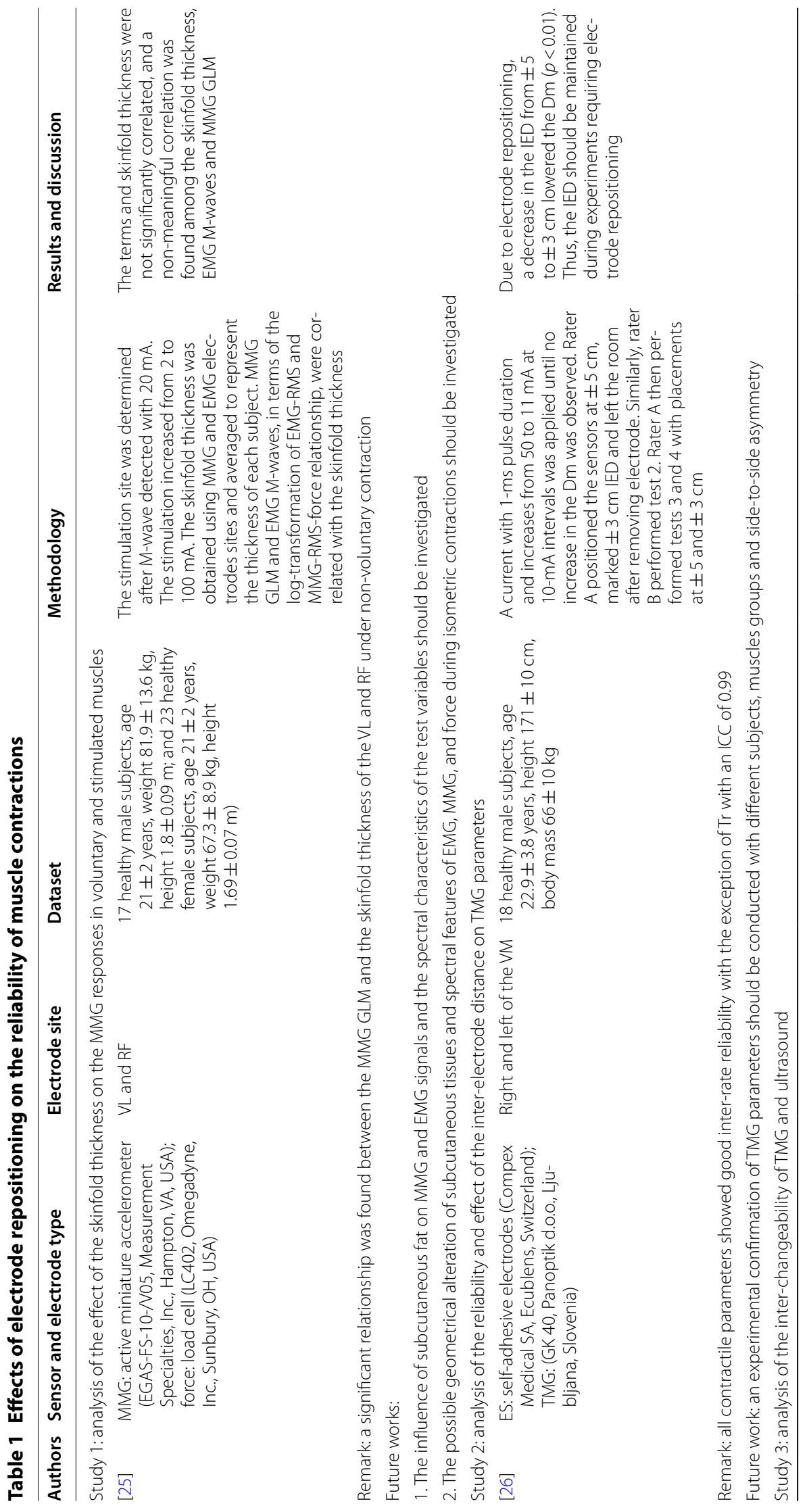




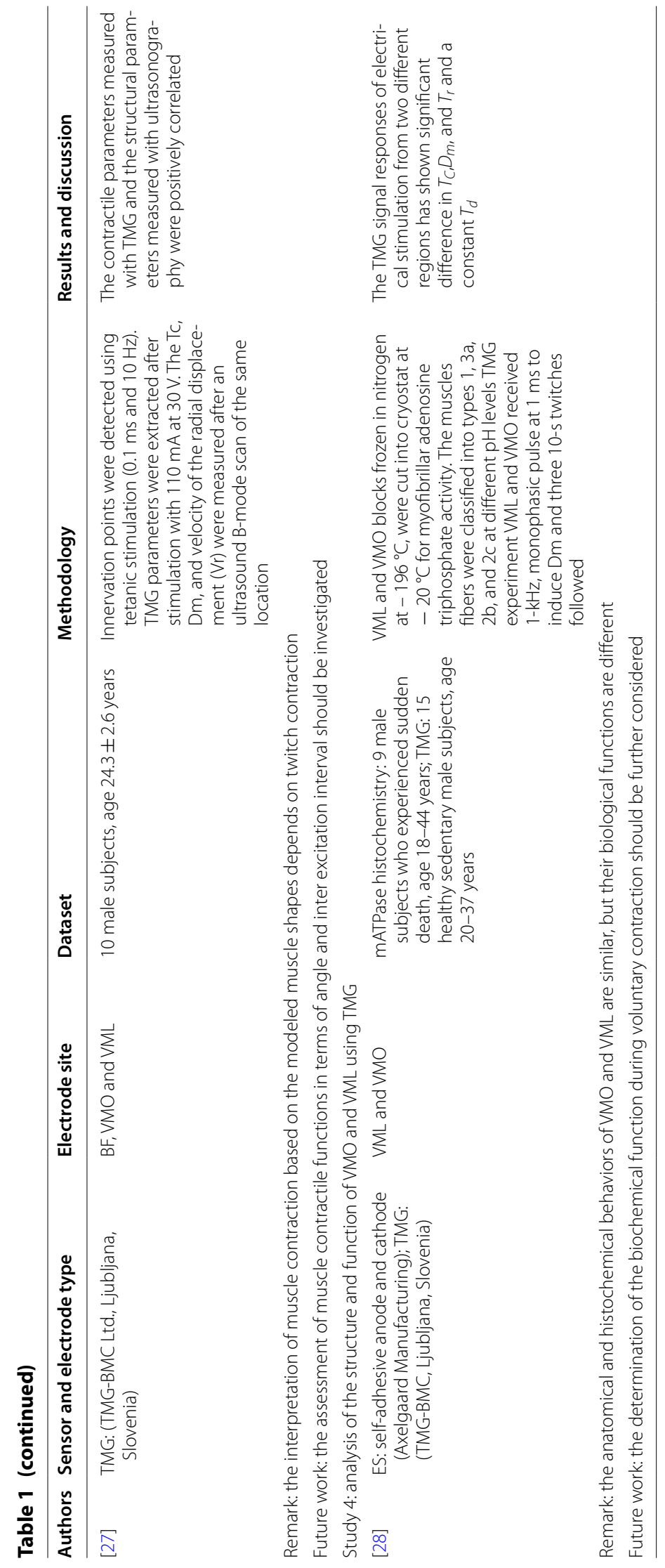


pennation angle were measured, and TMG signals were then obtained at the same measurement site. The authors found that TMG was deemed reliable for the assessment of muscle degradation [27]. Besides, a previous study [28] has showed that the application of a similar twitch type to two separate regions yielded different values of contraction time $\left(T_{C}\right)$ values. Thus, TMG can be used to detect the structure and function of the VML and VMO and should be verified for other muscles.

The results from these studies on the reliability of muscle function assessment showed that the functionality of TMG to detect the small muscle displacement gave the impression to record muscle contraction at different sensors' positions. Besides, these studies support the contention that the muscle belly displacement signal is influenced by IED. Hence, the interval between sensor positions on muscles should be kept constant in the studies of muscle activities using their mechanics. Further investigation should also shed light on the TMG characteristics considering the changes in muscle dimensions and positions for different tasks and postures.

\section{Stimulation protocol and muscle responses}

As presented in Table 2, nine studies [29-37] have been determined to examine the effects of the duration of the stimulation pulse on the muscle contractile properties. Some researchers [29] showed that the application of a percutaneous neuromuscular stimulation to the gastrocnemius medialis (GM) between 300 and $500 \mu$ s resulted in the displacement of the lateral belly and fixed contractile properties. The authors have recommended that the effects of skin impedance and other physiological moderators should be further examined. In another previous study [30], Yoichi Ohta evaluated the effect of nonisometric muscle contraction on joint kinematics and its reliability for detecting MMG responses. In this particular study, it was found that increases in the inter-pulse intervals or the number of stimuli yielded MMG signals that exhibited poor correlation with the changes in the excursion and angular velocity. Similarly, another study [31] revealed that the inter-day and inter-stimulus interval and the joint angle have altered the Dm, sustain time, and delay time, even though the obtained TMG parameters were deemed reliable.

The literature also demonstrates that the NMES frequency does not correlate with the MMG frequency response. Papcke et al. [32] reported that during NMES at 5\% maximum voluntary isometric contraction, the application of a 20-, 25-, 30-, 35-, 40-, 45-, 50-, 75-, and 100-Hz stimulation frequency did not correlate with any of the three axes analyzed during the Cauchy wavelet-based frequency analysis of MMG signals from the RF. Specifically, the mechanical characteristics of the RF have exhibited a frequency of $20-25 \mathrm{~Hz}$ that differs from and is not governed by the stimulation frequency. Hence, this study revealed that a high frequency does not impact the MMG characteristics and, therefore, does not affect the application of MMG in neuroprosthetics.

Postactivation potentiation (PAP) has been determined to be dependent on the joint angle and fiber composition. Specifically, a previous study [33] found a difference in the inter-muscle MMG due to PAP of the SOL and GM muscles at the ankle joint. After MVC in the neutral and dorsiflexion directions, a single stimulus was delivered, and a recording uniaxial accelerometer was positioned between two distal and proximal EMG electrodes on each muscle belly. After applying a stimulation 


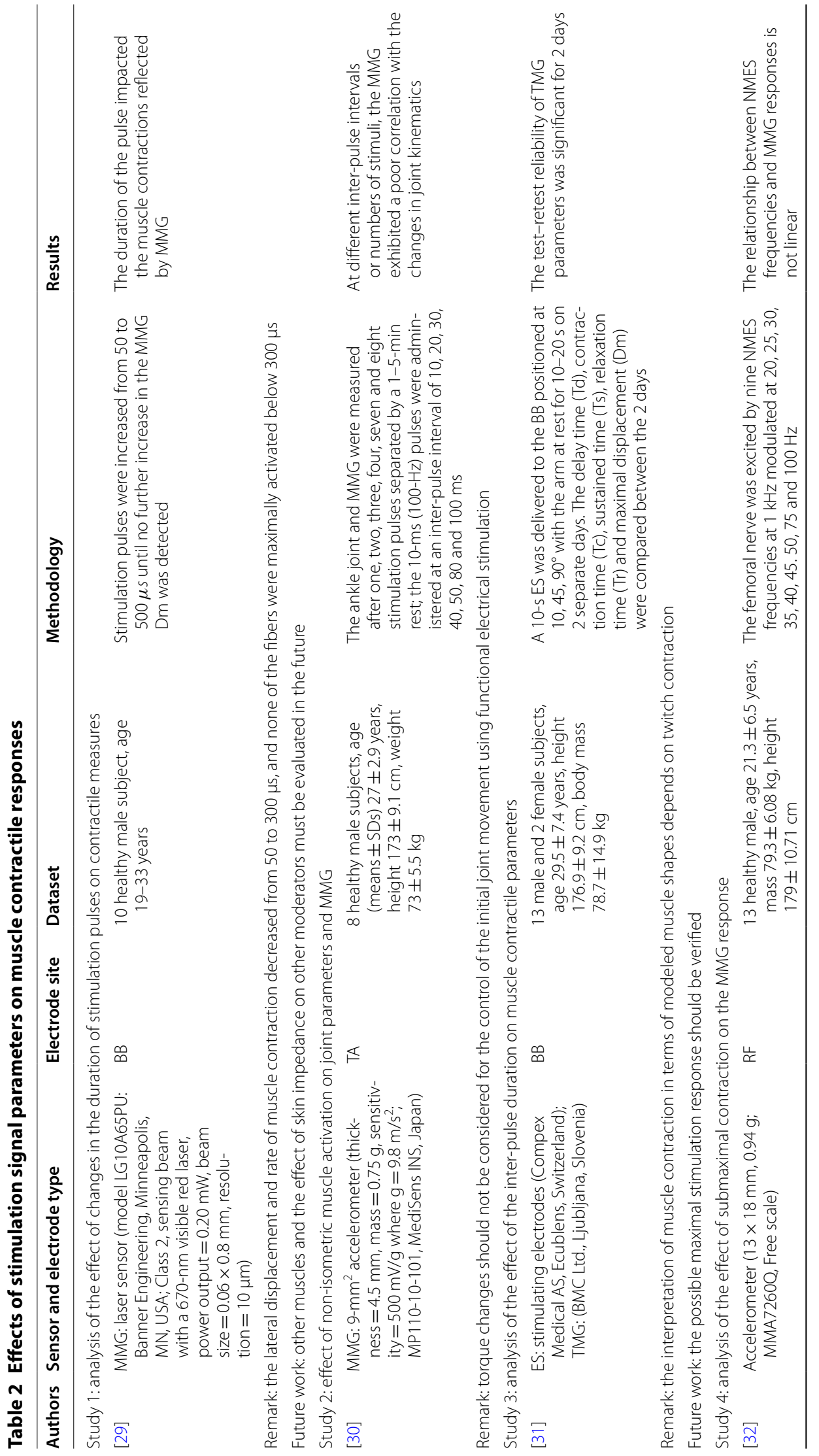




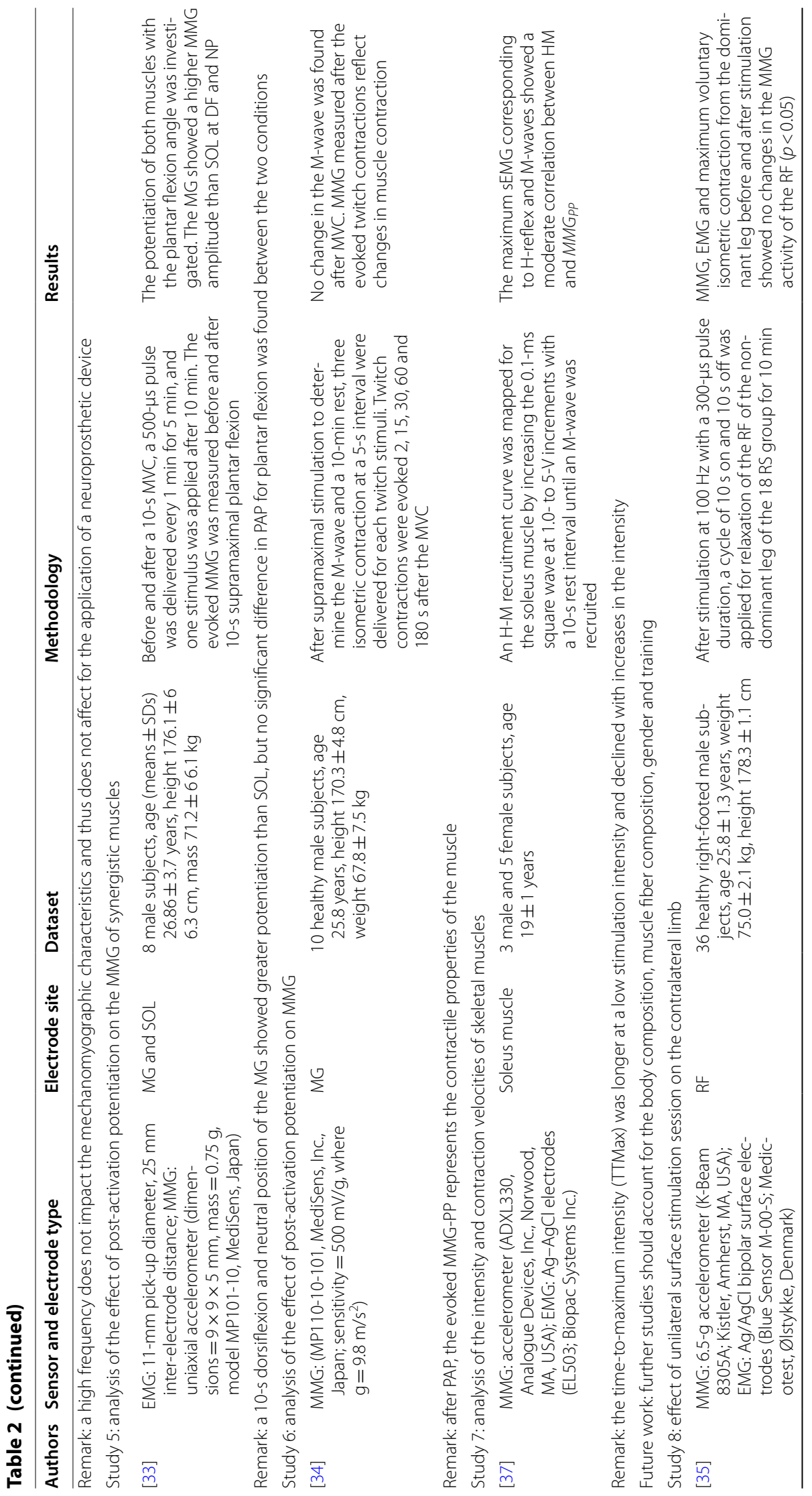




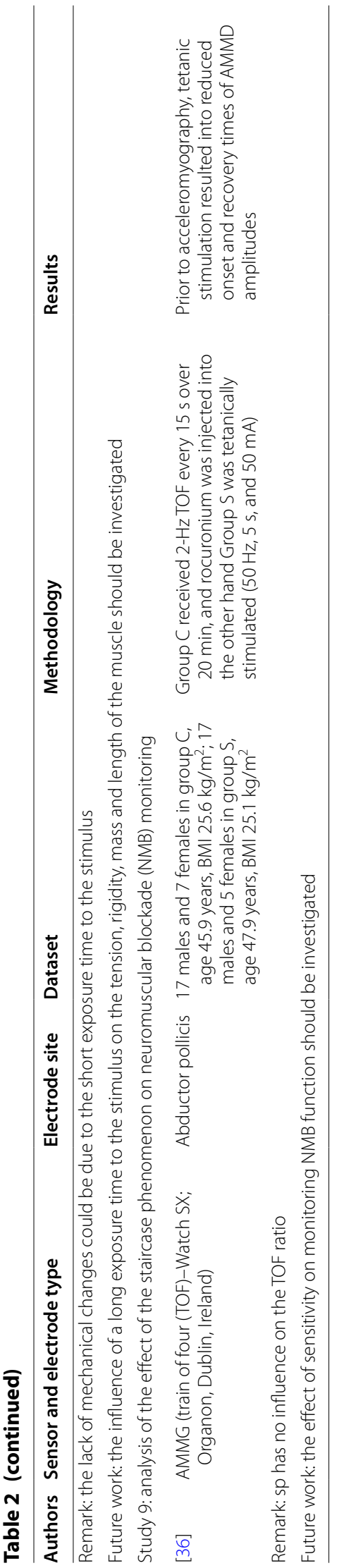


protocol similar to that applied in pre-MVC, simultaneous and individual MMG measurements yielded a higher peak-to-peak MMG amplitude from the GM than the SOL in the neutral and dorsiflexion directions. As both muscles were potentiated, it was determined that the magnitude of the PAP depended on the joint angle and composition of fiber types. Another previous study [34] used percutaneous neuromuscular electrical stimulation of the tibial nerve and twitch torque to evaluate the MMG and EMG responses of the GM. Thus, a positive correlation was found for the $\mathrm{MMG}_{\mathrm{pp}}$ with both $\mathrm{pT}$ and acceleration of twitch torque development $\left(\mathrm{d}^{2} \mathrm{~T} / \mathrm{dt}^{2}\right)$. Moreover, it was determined that the evoked MMG signals mirror the PAP force and that the mechanical changes in the muscles are deemed related to an increase in the twitch contraction. These findings reveal the reliability of MMG in characterizing the contractile properties of a muscle after PAP. Moreover, another study [35] has noted a stable MMG response before and after electrical excitation of non-dominant RF, despite increases in the EMG response and strength. In this particular study, it was suggested that a high exposure time can improve the mechanical behaviors. Meanwhile, another study [36] has evaluated the onset time, recovery time, and rate of changes in piezoelectric myographic amplitudes after a tetanic stimulation of the ulnar nerve, wherein reductions in the onset and recovery times were observed prior to measured myograph.

Specifically, the wavelet-based intensity analysis was reported to provide insight into the spectral characterization of individual muscle contractile properties. Using both MMG and surface (sEMG), the correlation between the MMG and H-reflexes was determined to support the hypothesis that muscle mechanics may provide insight into muscle recruitment strategy. The authors suggest that future work should evaluate the clinical implication of the MMG signals corresponding to H-reflexes and M-waves [37].

Insights from the reliability of muscles recruitment and function monitoring have shown that the stimulation pulse duration [29], number of stimuli [30], pulse rate [31], and joint angles [28, 34] provide global insights for elucidating the relationship between muscle contraction and recovery of their mechanical properties. Furthermore, the muscle fiber types and joint angles have been identified as two of the factors affecting the PAP [34]. Because MMG provides useful information on the musculoskeletal system, various MMG features, such as the onset time, recovery time, and relaxation time, should be examined after PAP [36, 37]. In contrast, no correlation was found between the frequency of stimulation and the MMG response [32]. Based on this perspective, the reliability of muscle recruitment and mechanical features remains an issue that should be addressed in future studies. In addition, the tissue underlying the skin, viscoelastic properties, and motor tendon units could alter the behaviors of the MMG signal. Therefore, muscle excitation and feature extraction techniques should be verified for output repeatable measurements. Interestingly, the variable and fixed contractile properties of the BB muscle's belly for known stimulation pulses were observed. Hence, a new parameter, namely, $\mathrm{M}+\mathrm{H}$, from the wavelet intensity analysis using both MMG and sEMG through NMES coupling encourages further verification of the technique for muscle recruitment using the stimulating signal pulses duration and frequencies of different muscles. 


\section{Studies on the electrodes for NMES and sensors for MMG measurement}

As shown in Table 3, six studies [38-43] have been determined to discuss the performance of developed electrodes and sensors in MMG and electrical muscle stimulation settings, further describing their ongoing development. In one study [38], a silver (Ag)polydimethylsiloxane (PDMS) composite was developed and used for electrical muscular activation. After fabrication, single and array electrodes were then used to excite the BB muscle. Their functionalities have been evaluated by comparing the MMG signals detected after electromyostimulation of the $\mathrm{BB}$ using the newly developed single and array electrodes to the ones detected using commercial NMES electrodes. As per the results, it was shown that the MMG signals obtained with single rather than array electrodes appeared to have a higher range. As such, ES of a specific muscle by single AgPDMS electrode is deemed more effective than array electrodes. Thus, single electrode yields a better signal-to-noise ratio compared with array electrodes. Array electrodes, on the other hand, have been determined to be crucial for wearable devices, particularly in cases in where nonuniform contact region could be present and where continuous NMES is needed.

Another previous study [39] has evaluated the applicability of a galvanometer-attached lever and skin indentor in MMG signal detection. During isometric contraction of the calf muscles, a dynamometer was used to record the surface response $3 \mathrm{~cm}$ from the motor point of the GM muscle. The contraction force was then recorded by a load cell. After the application of single-twitch electric intensity, an accelerometer was utilized to detect the target contraction on the skin surface. During evaluation of the effect of indention and muscle contraction, the amplitude RMS showed a proportional increase during contraction; furthermore, constant mean frequencies were observed. The accelerometer and galvanometer amplitudes were then preserved at $200 \mathrm{~N}$, but their double differentiation output reportedly decreased by $9 \%$. A unity cross-correlation coefficient confirmed the validity of using a galvanometer for recording MMG signals.

Interestingly, the availability of different forms of MMG recording devices has encouraged the examination of the validity of a micromachined acceleration sensor for TMG [40]. Simultaneous recordings, coupled with a linear optical encoder-based displacement sensor and accelerometer, were obtained to acquire the muscle belly displacement at 100 data points per $1 \mathrm{~s}$; in this experiment, a bandwidth of $2300 \mathrm{~Hz}$ was observed after a single-twitch square-wave stimulus of the BB. Based on previous studies, the researchers found that the results agreed with the minimal allowable threshold amplitude and a window length of $1 \mathrm{~mm}$. The standard deviation of the double integration of the linear displacement MMG increased over time. The mean relative error, the maximum displacement $(\mathrm{Dm})$, and $1 / 2 \mathrm{~T}_{\mathrm{r}}$ were $0.02 \mathrm{~mm}, 0.6$, and $3 \mathrm{~ms}$, respectively. Therefore, this study has confirmed that the microelectromechanical system (MEMS) accelerometer can be used to detect short-term small muscle displacement.

Meanwhile, another study [41] has examined the performance of displacement and acceleration transducers using a system identification method, with electrical stimuli as the input and acceleration (AMMG) or displacement (DMMG) as the output. Based on this study, it was determined that DMMG at a natural frequency of $3 \mathrm{~Hz}$ was suitable as a longitudinal mechanical feature, whereas AMMG was found to be of advantage when used for reflecting both the longitudinal and transverse mechanical characteristics 


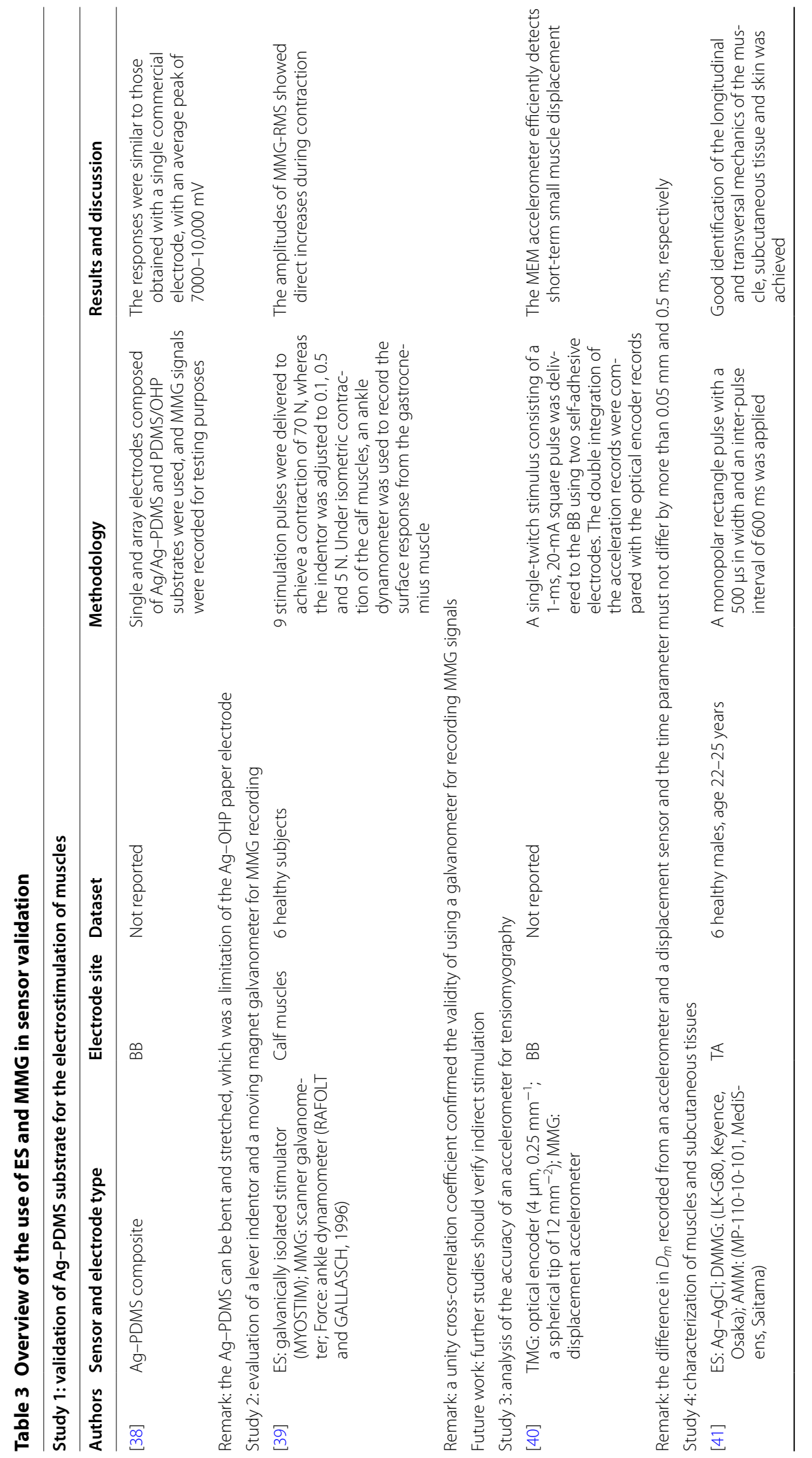




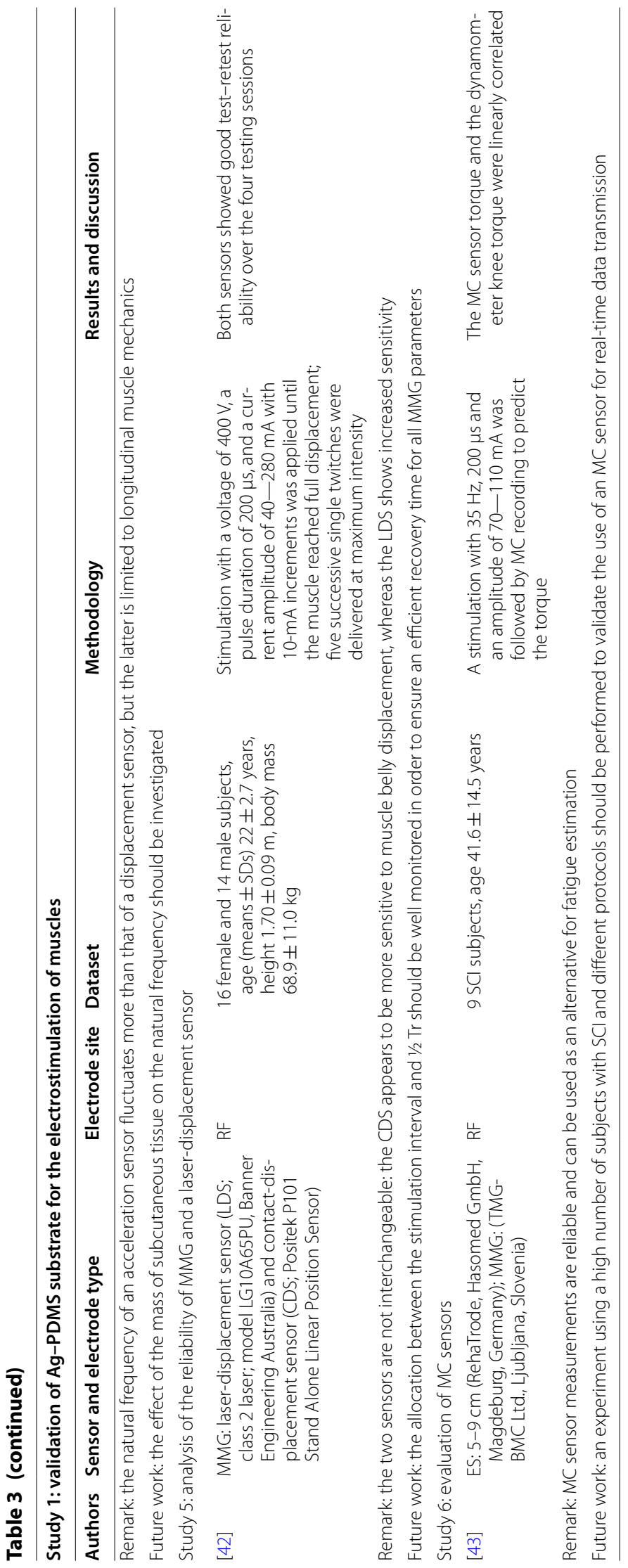


of muscles, subcutaneous tissue, and the skin. However, a 3-Hz higher fluctuation was noted for AMMG compared with DMMG. Although the sensors showed good performance in measuring underlying events, some evidence shows the importance of the transduction rate and sensitive parameters. In another previous study [42], it was found that a laser-displacement sensor (LDS) and a contact-displacement sensor (CDS) exhibited satisfactory reliability after four tests conducted over 2 weeks. Despite the recovery Tc and $D_{\max }$, the half-relaxation time $\left(1 / 2 \mathrm{~T}_{\mathrm{r}}\right)$ exhibited poor recovery to its pre-fatigued value during the recovery period. Despite the slower Tc of CDS, it was found that the sensor might detect individual muscle actions, whereas noncontact LDS exhibits some limitations, and this uniformity is thought to limit the inter-changeability of these sensors.

Meanwhile, another previous study [43] has compared the average and peak torques measured using a commercial dynamometer, with the torque estimated using a muscle contraction sensor (MCS) during functional electrical stimulation evoked muscle contraction. The results showed that the signals obtained using MCS and dynamometer were strongly correlated, which indicated that these sensors can be used instead. Although some limitations such as different responses of the LDS and CDS, AMMG and DMMG, effectiveness of VMG than MCS in muscle fatigue assessment for SCI population [44], there exist proof that confirms their validity in the myographic signal recording. Based on recent insights on selecting MMG sensors [45, 46], experimental verification with similar conditions and recording sites may provide better conclusions. A comparison of MMG recorded using MEMS accelerometer for TMG measurement showed that the MEMS accelerometer is reliable in terms of detecting small muscle displacement. AMMG was also found to measure both longitudinal and transverse muscle characteristics and subcutaneous tissues. In addition, the lack of fast recovery of $1 / 2 \mathrm{Tr}$ in LDS and slower CT response in CDS appear to be somewhat favor accelerometers as MMG sensors. Other available sensors such as piezoelectric sensors and MCS require further comparisons with MEMS accelerometers.

\section{Fatigue assessment}

In total, 18 of the 64 records have been identified to describe the examination of muscle fatigue using MMG and NMES protocols. Among these 18 studies, 10 articles in Table 4 utilized various techniques for the quantification of fatigue, 4 articles in Table 5 documented fatigue and muscle physiology, and 4 articles in Table 6 reported the relevance of fatigue and endurance. In one study [47], the development of fatigue was examined at $10 \% \mathrm{MVC}_{10 \mathrm{~min}}$ wrist extension during a 20 -min pre-experiment and its recovery over 10,30, 90, and $150 \mathrm{~min}$ under low-force contraction. The force analysis was conducted at 1,20, and $100 \mathrm{~Hz}$. The low-frequency fatigue (LFF) was measured as the response of the ratio of the $20-$ to $100-\mathrm{Hz}$ of stimulating signal. The $E_{M} G_{R M S}$ andMMG $G_{R M S}$ values were observed to increase at the force ratio of $20 / 100 \mathrm{~Hz}$ at $10 \%$ maximum MVC within 10 and 30 min of recovery. No significant changes were noted in the $80 \% \mathrm{MVC}$ test. In addition, no change in the mean power frequency (MPF) values was observed at $5 \% \mathrm{MVC}$, whereas a decrease before the experiment at 10\% MVC and after 90-150 min was detected. Therefore, these 


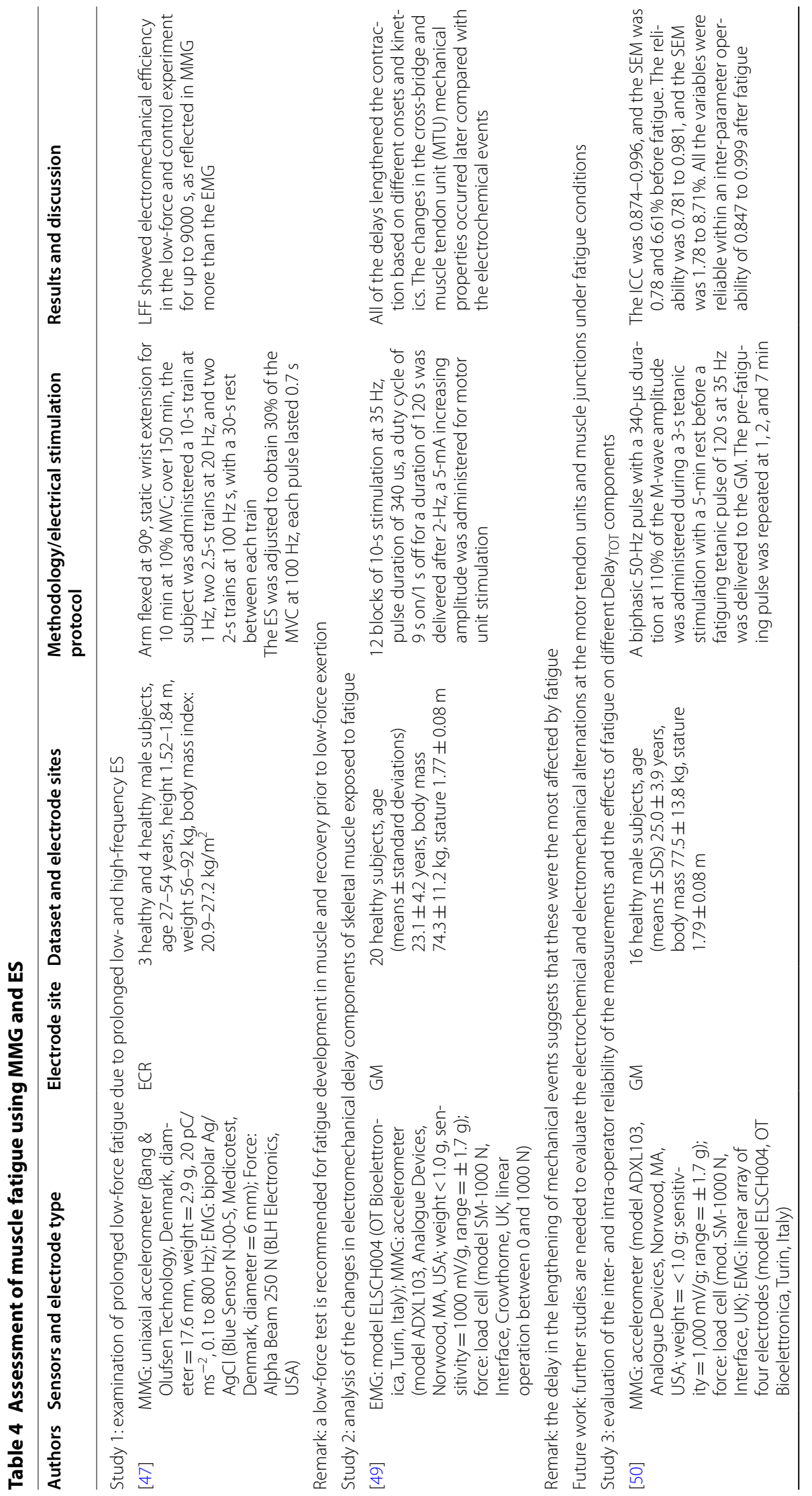


Uwamahoro et al. BioMed Eng OnLine $\quad$ (2021) 20:1

Page 18 of 47

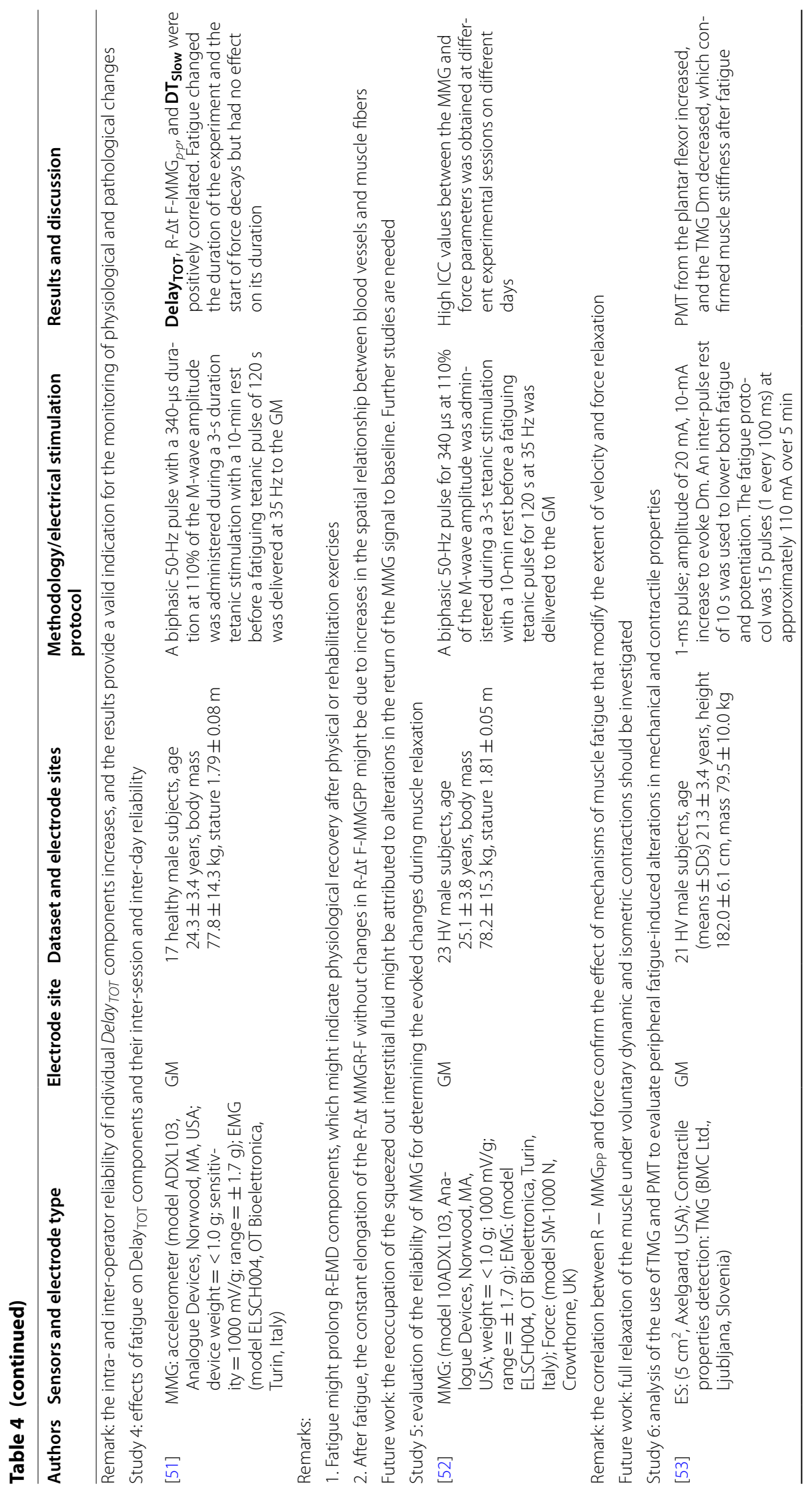


Uwamahoro et al. BioMed Eng OnLine $\quad$ (2021) 20:1

Page 19 of 47

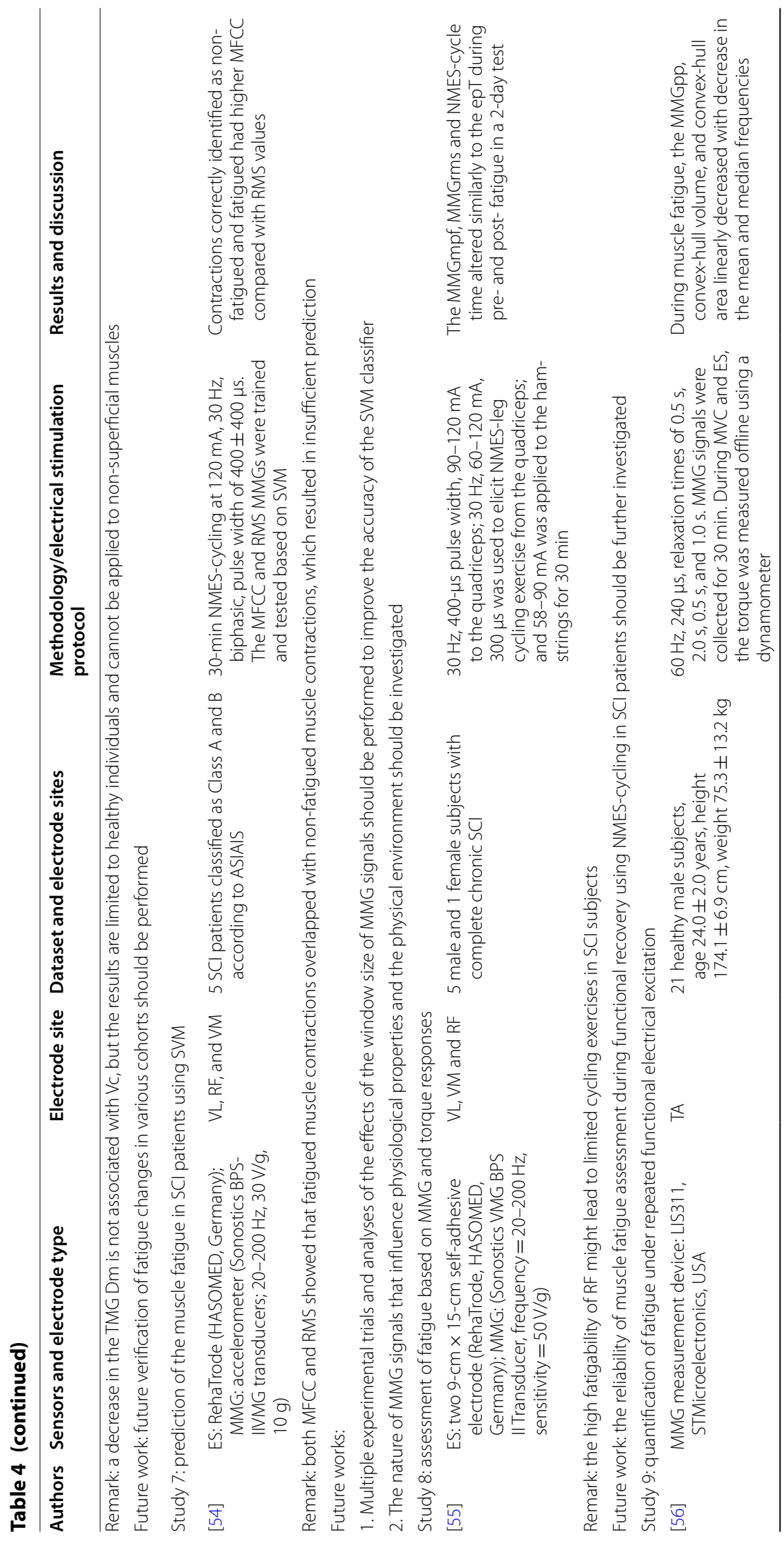




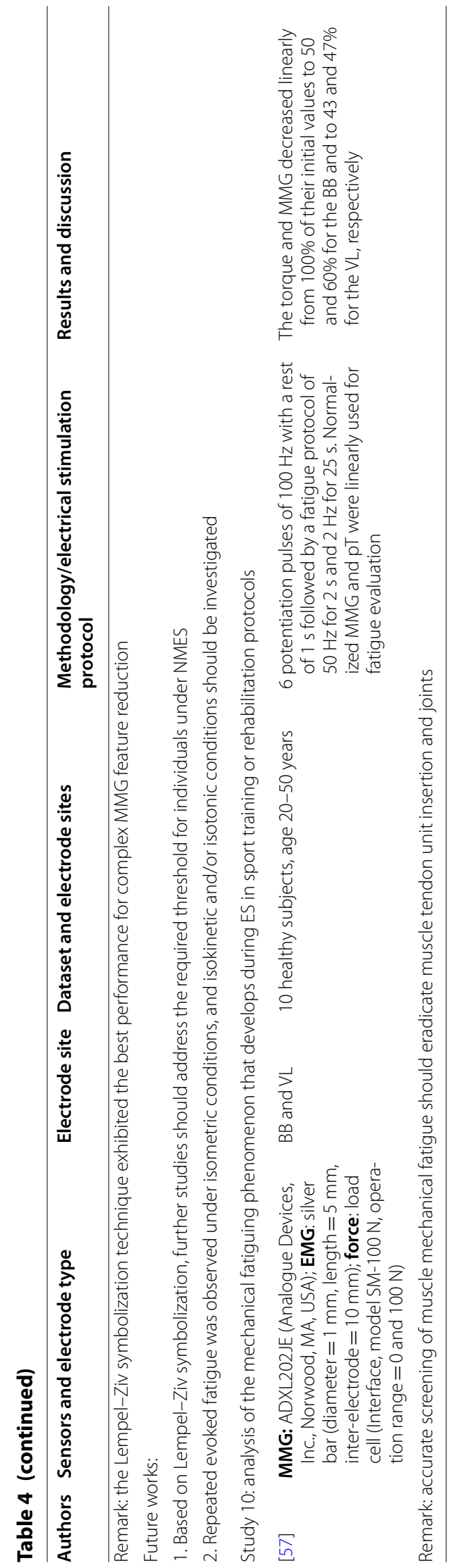




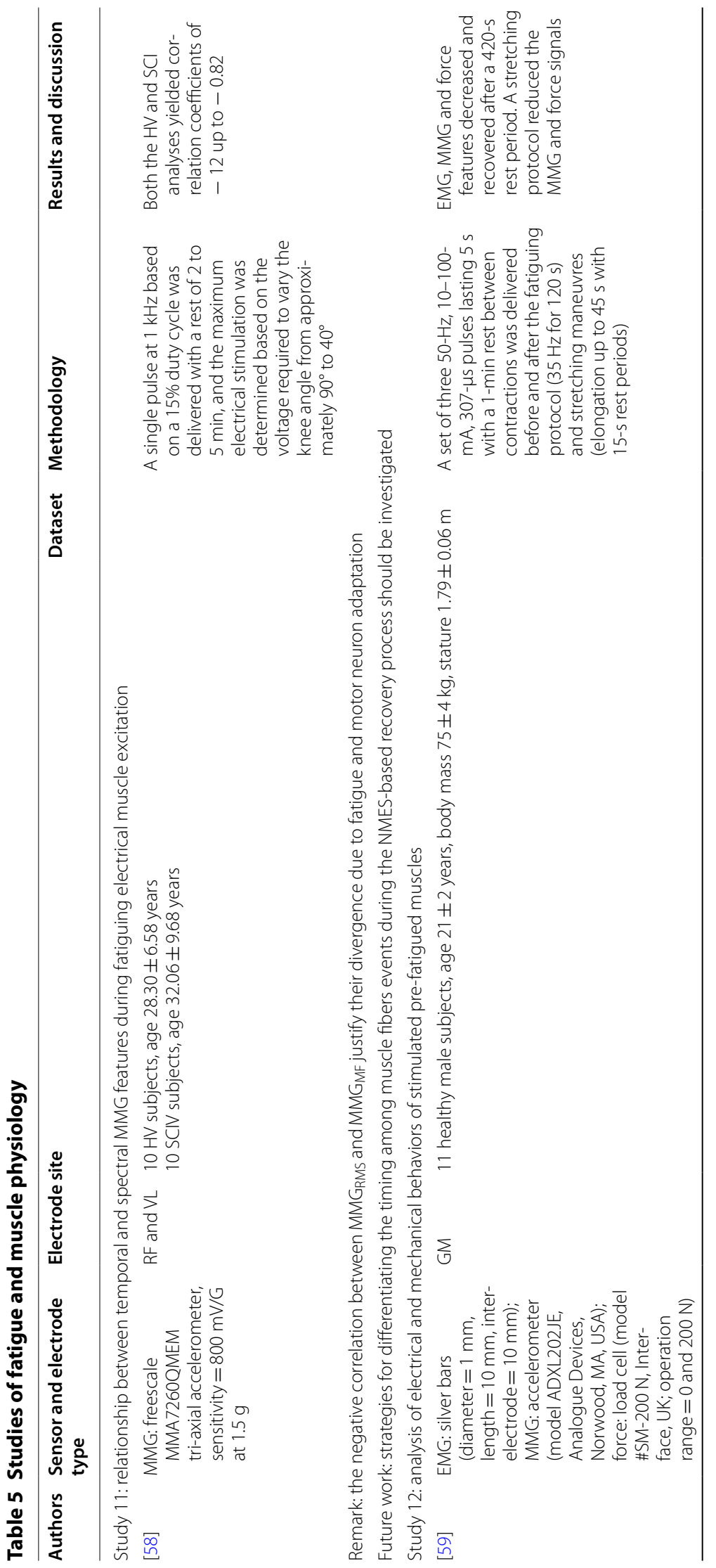



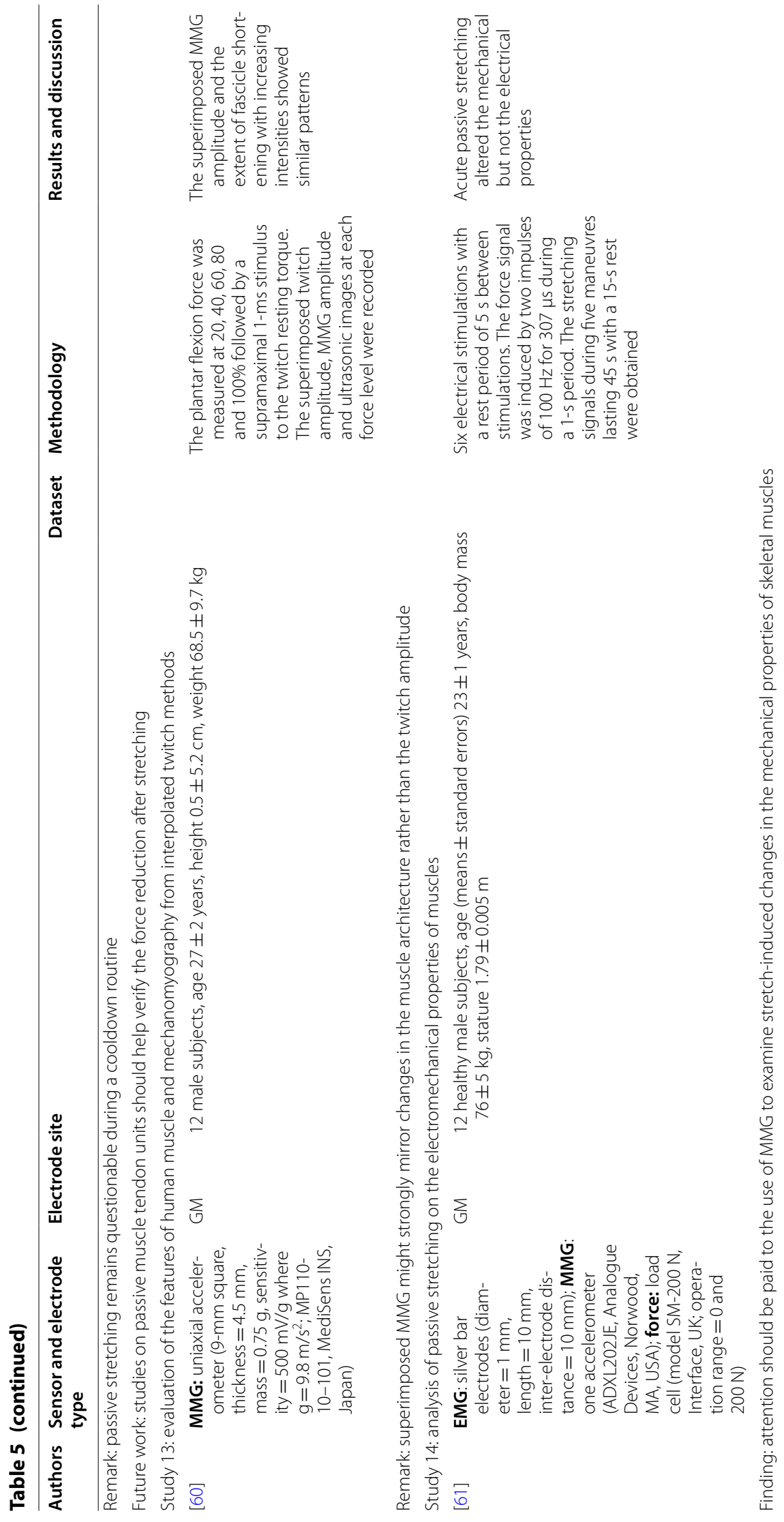


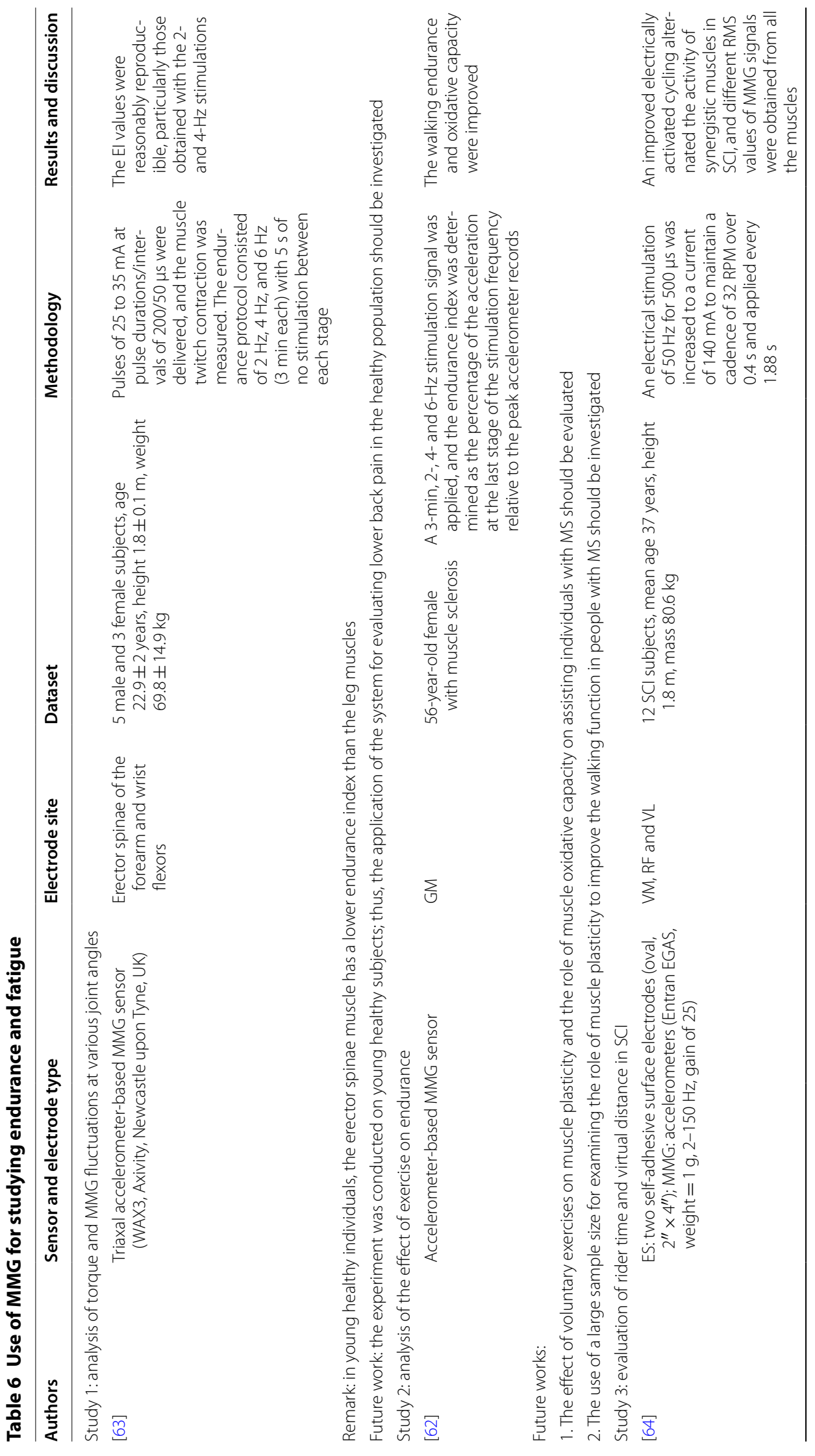




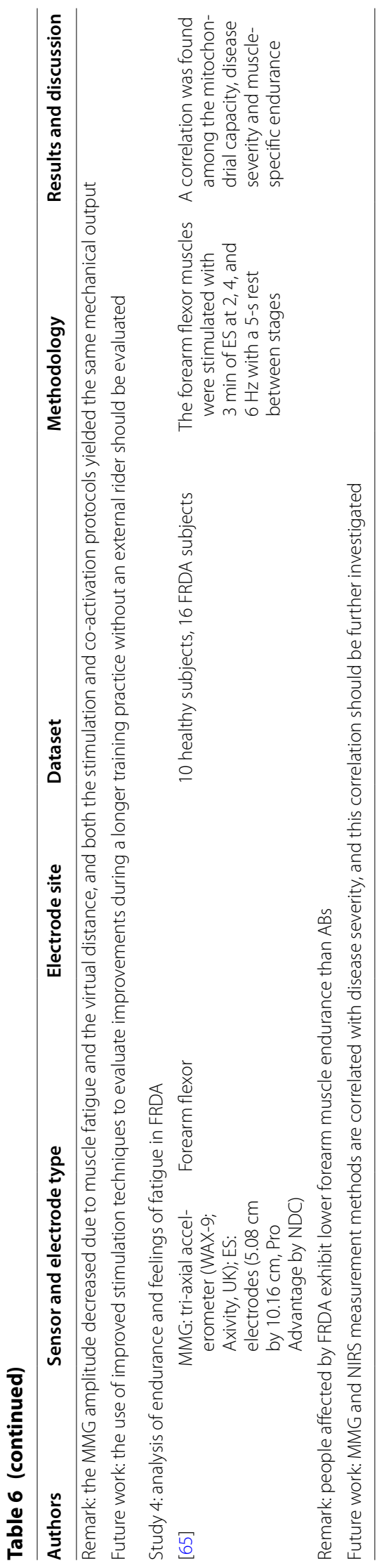


findings reveal that low-force muscle contraction leads to prolonged LFF, as identified by EMG and MMG. These results are supported by [48] where LFF was observed for $2 \mathrm{~h}$ after a $5 \% \mathrm{MVC}$ hand grip for $30 \mathrm{~min}$.

Meanwhile, four studies [49-52] have evaluated muscle behavior after fatiguing stimulation for $120 \mathrm{~s}$, followed by tetanic stimulation at 1,2, and $7 \mathrm{~min}$. One of these studies [49] evaluated the changes in electromechanical delay components from the GM. The Delay $_{\text {TOT }}$ values were determined based on the RMS and MF of the EMG, MMG, and force. All measurements were obtained from a time frame of $250 \mu$ s during fatiguing stimulation. During a 120-s stimulation, $\mathrm{MMG}_{\mathrm{RMS}}$ was noted to show stable patterns over the first $90 \mathrm{~s}$, but then decreased until the end of the stimulation. The MF of MMG presented a strong reduction during the first $10 \mathrm{~s}$; then, it monotonically decreased until the end of stimulation. In contrast, the EMG $\mathrm{RMS}_{\mathrm{S}}$ increased by $40 \%$ during the first $70 \mathrm{~s}$ before it returned to its initial values, whereas the MF components presented reductions starting after the first $10 \mathrm{~s}$ until the end of the stimulation. In this study, various electrochemical and mechanical components were identified, and the researchers reported that the detected delay correlated with the tested EMG, MMG, and force parameters. In addition, the researchers claim that the mechanical events ( $\Delta \mathrm{t}$ MMG-F) provided a highly reliable measure of fatigue. These findings were consistent with those obtained in another study [50] that investigated the effect of fatigue on the delay in force development (Delay ${ }_{\text {TOT }}$ ) from GM during $35-\mathrm{Hz}$ fatiguing stimulation for $120 \mathrm{~s}$. Before and after the induction of fatigue, a couple of tetanic stimulations were administered, with a resting interval of $10 \mathrm{~min}$, and the effect of fatigue was indicated by the reductions in $p^{\mathrm{F}}$ and $\mathrm{MMG}_{p-p}$. The results also showed that fatigue lengthened the delay, which in turn affected its electromechanical and electrochemical components.

Similarly, another study [51] has examined the impacts of fatigue on electromechanical delay components and assessed the inter-day and inter-session reliability during relaxation after tetanic and fatiguing stimulation protocols, same as those used in the above-mentioned study [50]. The results demonstrated that the increase in $\mathrm{R}$ - Delay тот paralleled the increase noted in electrochemical components and the first mechanical component measured after fatigue, whereas the second mechanical component, which was an electromechanical component measured at the onset of the force decay and the negative MMG peak values, did not change. Therefore, a low contribution to the $\mathrm{R}$ - Delay TOT $_{\text {was found. }}$

The aims of another study [52] were twofold: (1) to examine the correlations between peak-to-peak MMG and other force parameters and (2) to validate the interday reliability of mechanical parameters of the MMG signals induced by a tetanic stimulation before and after the NMES to fatigue. The authors reported that $p^{\mathrm{T}}$, MMGpP, $\mathrm{R}-\mathrm{MMG}_{P-P}$, and the acceleration of force development, the slope, and relaxation decreased after fatiguing stimulation, but the relaxation time (RT), CT, and $\tau$ were noted to increase. The relaxation MMG $\left(\mathrm{R}-\mathrm{MMG}_{P-P}\right)$ correlated with the $\mathrm{MMG}_{P-P}$, slope, $\mathrm{\tau}, p^{\mathrm{T}}$, and the acceleration of force development $\left(\mathrm{D}^{2} \mathrm{RFD}\right)$ before and after fatigue. Due to the high intraclass correlation coefficient between the MMG and force parameters obtained from different experimental sessions on different days, it was concluded that the MMG is a viable alternative to force in terms of examining fatigue-induced changes during muscle relaxation. 
Furthermore, Macgregor et al. [53] have examined muscle fatigue based on muscle tension through the temporal and spatial displacement recorded from GM before and after supramaximal stimulation through a process known as TMG. As per the result of this study, a significant $(P<0.001)$ decline was noted in the peak force measured with MVC; thus, it was hypothesized that this decline was in line with the decrease in the TMG Dm $(P=0.031)$. Similarly, the researchers claimed that the passive muscle tension from the plantar flexor increased.

In a previous study [54], the mel frequency cepstral coefficient (MFCC) and $M_{M} G_{R M S}$ were used to train an SVM classifier for the identification of fatigued and non-fatigued RF, VL, and vastus medialis in SCI. MFCC were then obtained as its time-localized frequency information using short-time Fourier transform of a stationary signal with a window frame of $25 \mathrm{~ms}$. The MFCC features exhibited 90.7\% accuracy, whereas the RMS feature only showed $74.5 \%$ accuracy.

Meanwhile, three studies [55-57] have also examined muscle fatigue via analyzing torque and MMG profiles. One of these studies [55] assessed the MMG responses from the quadriceps muscles to quantify its changes between pre- and post-fatiguing conditions in SCI patients in NMES-evoked cycling. The peak torque and normalized $M M G_{R M S}$ and $M M G_{M P F}$ values showed significant trends between pre- and postelectrical excitation for individual muscles. The researchers also observed a change in the MMG signals as a function of the cycling time for the VL, RF, and VM muscles. Typically, $\mathrm{MMG}_{\mathrm{RMS}}$ and epT significantly $(P<0.05)$ decrease as the leg cycling exercise increases for the quadriceps groups.

On the other hand, Massimiliano Gobbo and colleagues [57] have investigated the validity of MMG to assess the fatigue that develops during NMES in rehabilitation services. After a fatiguing stimulation of 50 single twitch and $2 \mathrm{~Hz}$ for $25 \mathrm{~s}$, a correlation was found between the mean values of normalized $p^{\mathrm{T}} \%$ and MMGPP $\%$. It was further reported that $p^{\mathrm{T}} \%$ and $\mathrm{MMG}_{\mathrm{PP}} \%$ decreased from $100 \%$ of their initial values to 50 and $60 \%$ for the $\mathrm{BB}$, respectively, and to 43 and $47 \%$ for the $\mathrm{VL}$, respectively. In addition, the decreases in $p^{\mathrm{T}} \%$ and MMGPP $\%$ exhibited a different linear correlation. Thus, the introduction of electrical activation of skeletal muscles and MMG responses into assistive technology that involves analysis of torque of a particular muscle remains to be unclear.

Under both artificial electro-muscle excitation and MVC, Jo et al. [56] examined the fatigue from the ankle joint and performed a torque analysis. The raw MMG data were then extracted during $30 \mathrm{~min}$ of repeated stimulation of the tibialis anterior (TA) muscle, sampled at $1 \mathrm{kHz}$ and bandpass filtered at 8-100 Hz. The amplitude, i.e., convexhull, peak-to-peak, and Lempel-Ziv algorithm, and median and mean frequency-based MMG features were also utilized for the quantification of fatigue of the TA muscle. The ankle joint torque has been used to analyze fatigue after low-pass filtering of the raw torque at a cutoff frequency of $3 \mathrm{~Hz}$. The muscle fatigue was further indicated by a linear decrease in torque during consecutive stimulation patterns. The coefficient of determination $\left(r^{2}\right)$ was 0.7823 . However, it was noted that the frequency components of MMG signals demonstrated a weak linear relationship with fatigue. Thus, the use of the stimulation protocol relevant to NMES popular in clinical settings supports the finding that the technique may fit the design of electrical muscle excitation feedback control system, which is useful in assisting week/injured muscles. 
Four studies [58-61] have been identified to discuss the physiological behaviors after fatigue administration. In one of these studies [58], the authors evaluated the correlation between the temporal and spectral features of MMG from $10 \mathrm{SCI}$ and 10 health volunteers undergoing recovery by NMES.MMG $\mathrm{RMS}_{\mathrm{S}}$ and $\mathrm{MMG}_{\mathrm{MF}}$ from the analysis of window length were examined. An increase in RMS and a decrease in MF were then observed from both groups. The authors concluded that the temporal features might originate from the increased amplitude of mechanical wave from motor unit coherence, whereas the decrease in MF might be attributed to motor unit adaptation.

On the other hand, two studies $[59,61]$ evaluated the effects of stretching on mechanical and electrical responses, wherein both analyses found constant electrical parameters but a divergence in the mechanical features. One of these studies [59] used a stretching protocol in order to evaluate the changes in the mechanical and electrical properties of EMG and MMG on GM. Significant decreases in the RMS and MF of EMG have been noted, and a conduction velocity was observed after the fatiguing protocol. Similarly, the RMS and peak-to-peak values of MMG were noted to decrease, whereas the speed of electromechanical impulse and the $1 / 2 \mathrm{~T}_{\mathrm{r}}$ increased. The authors claimed that all RMS values of EMG and MMG returned to their pre-fatigued values after stretching, whereas the peak rate of force development, derived acceleration, and the $1 / 2 T_{r}$ showed further reductions. This led the researchers to a conclusion that the application of acute stretching to previously fatigued muscles weakened the mechanical but not the electrical properties.

In contrast, another study [61] has evaluated the electrical and mechanical manifestations of muscles after a set of six electrical stimulations before and after stretching. The findings are in line with those obtained in the above-mentioned study [59], revealing no significant changes in the EMG signal parameters. In addition, the study found reductions in the force acceleration, peak force, and peak-to-peak MMG values, and these reductions were determined to be accompanied by a possible concomitant decrease in muscle stiffness. Moreover, the researchers found a poor correlation between the MMG and force parameters, wherein a bout of acute passive stretching alters the mechanical but not the electrical parameters of electrically stimulated muscles.

Furthermore, the mechanical properties of skeletal muscles were also investigated in a previous study [60], with the aim of characterizing the architectural changes in the human MG under interpolation conditions. Using ultrasound images obtained from electrically excited MG under plantar flexion at 20,40,60, 80, and 100\% MVC, the researchers found that superimposed MMG amplitudes exhibited a curvilinear decrease accompanied by a fascicle shortening, with increases in the contraction intensity up to $100 \%$ MVC. In contrast, the study argued that the superimposed twitch amplitude decreased linearly with increases in the contraction intensity up to $80 \% \mathrm{MVC}$.

Four studies [62-65] have also reported on the use of MMG and fatigue as a reflection of muscle endurance. One study [62] aimed to diagnose the drawback of exercise-based improvements in disabled individuals. Assisted by electrical muscle activation, the walking speed and muscle endurance were compared to the working function and strength. As per the endurance index, the endurance was noted to increase with increases in the walking function in individuals with multiple sclerosis. Similarly, another study [63] highlighted the dependency of the endurance index on MMG recorded from electrical 
activation of the erector spinae muscle compared with those from the voluntary or muscle oxygen levels. Another study [64] has also used different stimulation protocols to examine endurance in SCI-paralyzed patients under different workloads. The authors reported that a high stimulation intensity, compared with the lower intensity, can lead to increased muscle fatigue and hypothesized that the standard co-activation stimulation protocols are less prone to cycling endurance time accounted in NMES-based functional recovery compared with alternative stimulation.

Meanwhile, a previous study [65] evaluated the endurance, feeling of fatigue, and mitochondria capacity using NMES, near-infrared spectroscopy (NIRS), and MMG at different age ranges. The forearm flexors of 10 abled bodies and 16 Friedreich's ataxia (FRDA) were electrically stimulated for $3 \mathrm{~min}$ at 2, 4, and $6 \mathrm{~Hz}$, with a resting interval of $5 \mathrm{~s}$. The MMG records indicated a considerable correlation in terms of mitochondrial capacity, disease severity, and muscle-specific endurance in FRDA. Based on these findings, it can be concluded that the disease frequency and progression in the FRDA population can be monitored.

It was indicated that MMG signals are obtained from muscles, which represent the different physiological conditions of those muscles through fatigue protocols. It is likely that a decrease in spectral component during functional recovery produced by NMES can be due to motor unit adaptation, whereas the increase in $M_{M M} G_{R M S}$ might be due to motor unit coherence. In addition, a decline in MMG parameters and torque with an increase in fatiguing NMES demonstrates the usefulness of electrical stimulation and muscle mechanics in determining muscle injury in SCI population. Hence, fluctuation in mechanical features manifested as a muscle response in different experiments requires further exploration. It was also pointed out that LFF might be attributed to low \% MVC. However, this was contradictory to other studies as this was identified to be due to the slower ability of muscles to recover their normal activity under low frequency or overlap between signals from damaged and fatigued muscles. The role of muscles mechanics has also been determined using MFCC, which is a feature extraction technique that uses speech signal processing and other nonstationary signals such as EMG classifications and electroencephalography (EEG) [66]. As detailed in the literature, the developed model exhibited good accuracy in muscle fatigue assessment. The features from MMG have also reported the correlations among specific muscle endurance, mitochondrial capacity, and diseases severity in FRDA population.

Collectively, the findings from these studies provide evidence that muscle fatigue should be re-investigated. Specifically, the overlap between muscle damage and fatigue, the decrease in the angle caused by muscle weakness, and the overlap between muscle endurance and disease severity and progression in populations other than the FRDA population should be further examined. Thus, muscle mechanics based on MMG has been experimented in electrical stimulation-based cycling exercises but requires further investigation in biofeedback control system required in assistive technology for the limbs rehabilitation.

\section{Force assessment}

As presented in Table 7, four studies [67-70] have been determined to examine the influence of muscle conditions on force and MMG recorded under NMES. One of these 

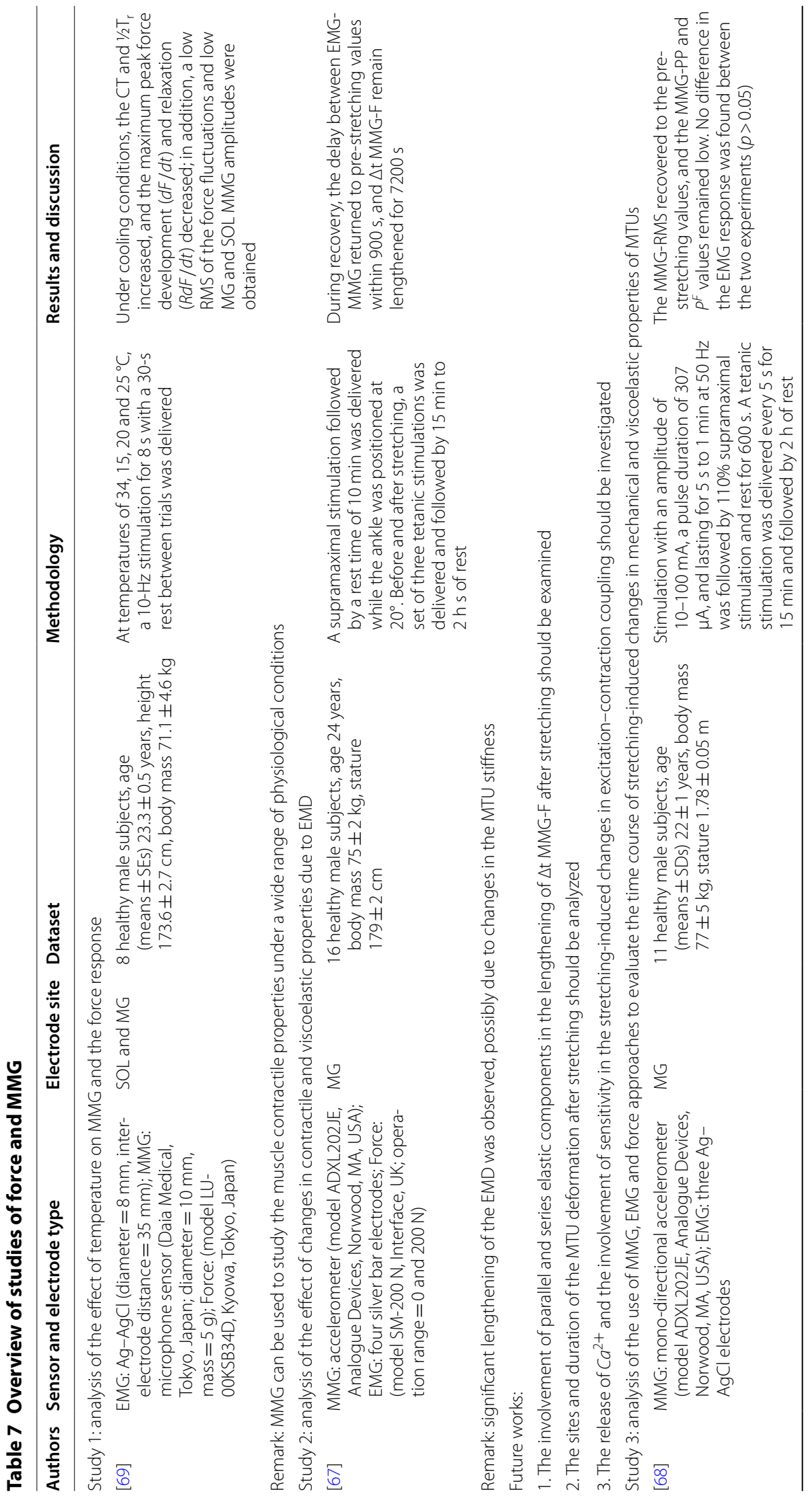


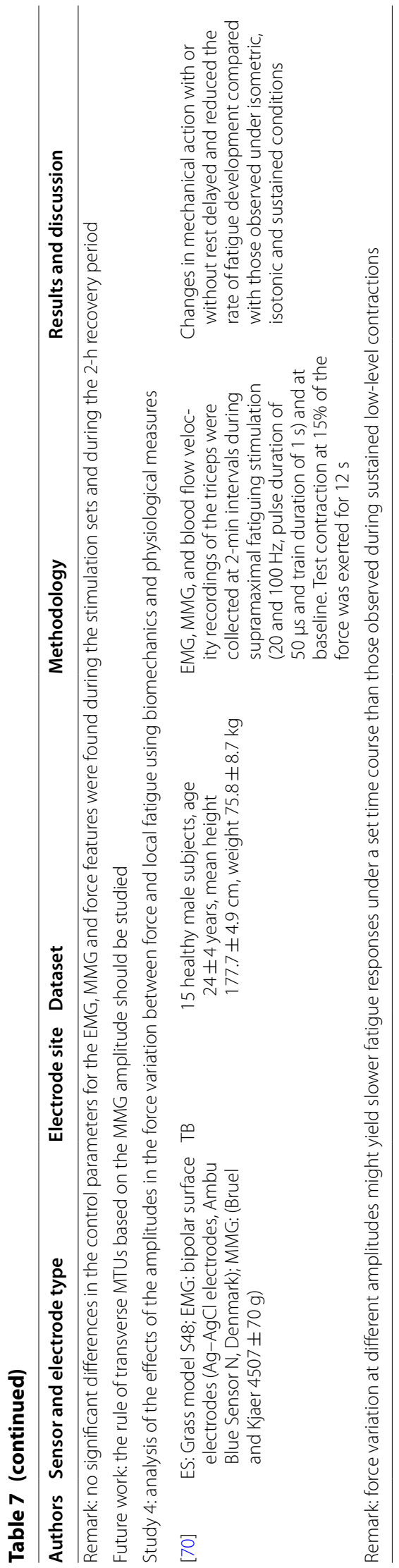


studies [69] has attempted to determine the effect of temperature on contractile parameters using temporal and spectral features of MMG signals. After electrical intensity was delivered to the GM and SOL muscles, the force signals and MMG responses were examined under a controlled temperature. The authors of this study then reported a decrease in the maximum peak force development and relaxation under cooling conditions. In contrast, the $\mathrm{CT}$ and $1 / 2 \mathrm{~T}_{\mathrm{r}}$ increased under hypothermia. Further, an increase in $\mathrm{M}$-waves was also noted, which justifies the decreases in the muscle conduction velocity. These findings encourage the use of MMG for screening the muscular contractile behaviors under varying physiological circumstances. The use of MMG-, EMG-, and force-based approaches to evaluate the time course of stretching-induced changes in the mechanical and viscoelastic properties of MTU was examined in $[67,68]$. The findings obtained in one of these studies [67] revealed that an increase in the EMD followed a reduction in $p^{\mathrm{F}}$. The time delay between EMG and MMG recovered to its initial value within $15 \mathrm{~min}$, whereas the delay between MMG and force value was maintained for $2 \mathrm{~h}$. These findings only confirmed the effect of stretching and encouraged the use of MMG, EMG, and force as indicators of the recovery of muscle properties after the induction of mechanical stress on the MTU viscoelastic properties. On the one hand, $p^{\mathrm{F}}$ and MMGPp did not exhibit recovery, and on the other hand, the short recovery observed in $M_{M} G_{R M S}$ indicated a recovery in the viscoelastic properties of parallel components after a short period [68]. Biomechanical and physiological responses were also used to evaluate the slowing of fatigue due to changes induced by occupational mechanical exposure. Using various force amplitudes, another study [70] applied electrical stimulation quantities to the triceps brachii (TB) at 15\% MVC to elicit LFF. Therefore, the subject performed MVC for $5 \mathrm{~s}$, and the force was recorded for $3 \mathrm{~s}$. The authors then concluded that (i) local fatigue might be reduced in individuals performing low-load tasks and (ii) muscle rest does not exert more marked effects than those predicted by variations in the force amplitude. These findings imply that changes in mechanical action with or without rest delay can reduce the rate of fatigue development compared with the development observed under isometric, isotonic, and sustained conditions. Therefore, it was suggested that muscle exposure to time-varying forces might reduce the degree of local fatigue during low-load tasks. The observations described in this review highlighted the relationship of muscle conditions, force, and daily tasks using MMG through NMES. Hence, muscles screening should take physiological conditions and daily occupational exposure circumstances into consideration.

\section{Assessment of muscle stiffness}

Using MMG and NMES, six studies [71-76] in Table 8 have been identified to examine muscle stiffness. One of these studies [75] has utilized evoked MMG signals from voluntary MMG and walking acceleration in order to estimate stiffness. Combined with the undamped natural frequency, the mass of the VL estimated as $0.99 \%$ of the subject's weight was also used in an attempt to estimate stiffness. The result was then compared with the stiffness estimated using the identification technique, wherein muscle stiffness was found to exhibit a prior relationship with both workload and power.

Furthermore, the analysis on the synchronous averaging of evoked MMGs in a previous study [71] was performed through identifying both longitudinal and transverse 


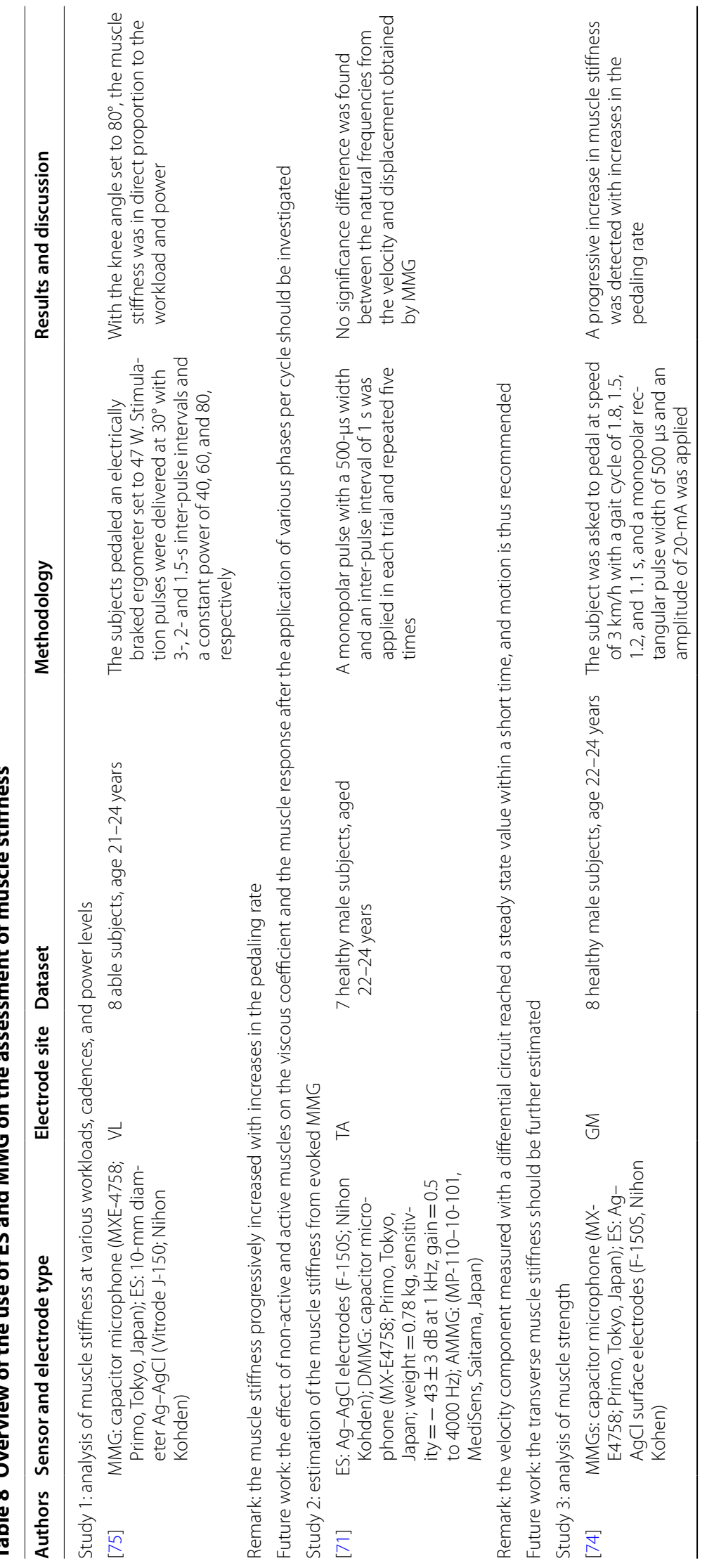




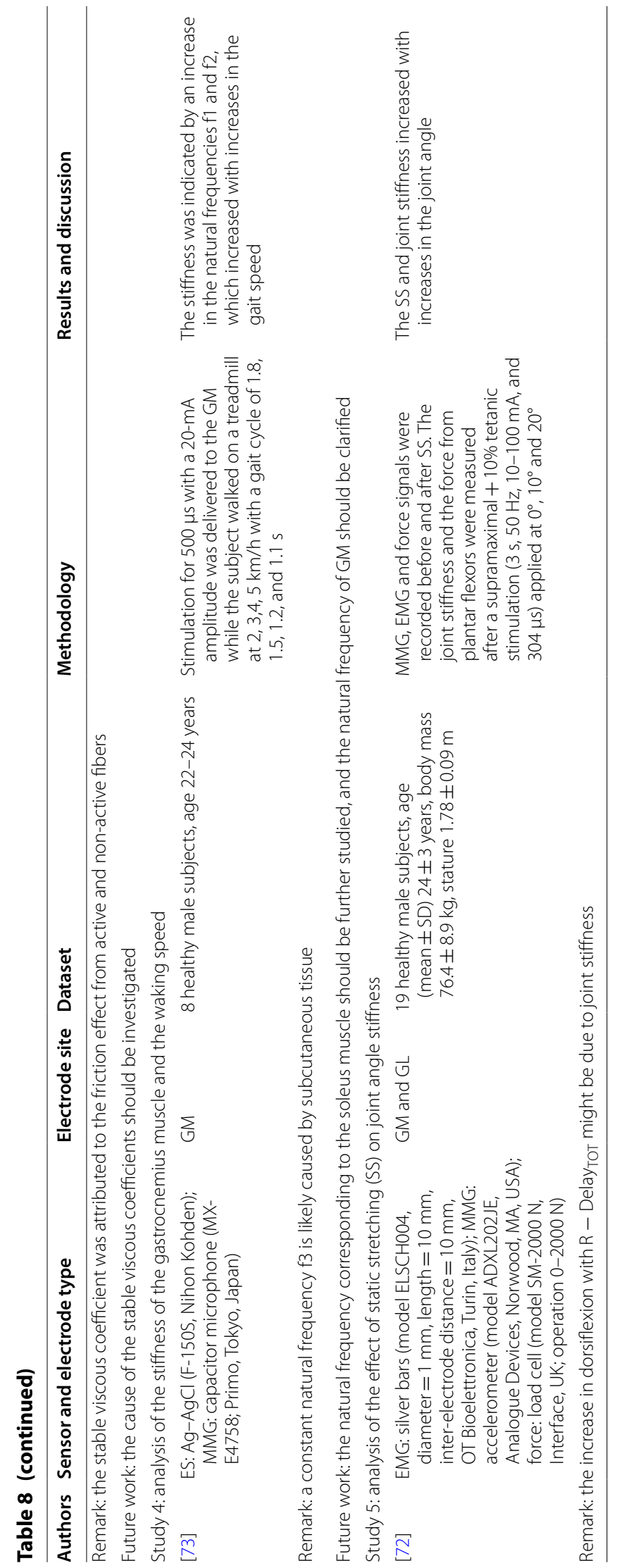


muscle stiffness from MMG signals obtained via electrical activation of the peroneal nerve. A capacitor microphone and a differential circuit were used for recording purposes. The stiffness was measured from a natural frequency, assuming that the mass of the TA muscle equals $0.2 \%$ of the participant's weight. In another study [76], the singular value decomposition (SVD) was used in determining the transfer function from stimulated to evoked MMG signals. The velocity MMG yielded two natural frequency components denoted as $\mathrm{f} 1$ and $\mathrm{f} 2$ : $\mathrm{f} 1$ mirrored the longitudinal stiffness, which was approximated to the values in the displacement and acceleration MMG systems, and $\mathrm{f} 2$ was determined from the velocity MMG and was found to be similar to the natural frequency of the acceleration MMG system. The resulting value was then regarded as the transverse muscle stiffness but was not estimated from MMG signals. The displacement and acceleration MMG yielded three natural frequencies, which were denoted as f1, f2, and $\mathrm{f} 3$, and no significant difference was determined between the natural frequencies from velocity and displacement MMG.

In the same study [76], electrical stimuli were transcutaneously delivered to the VL muscle while the participants were ask to pedal an ergometer. The mass of the muscle and the coefficient of the transfer function estimated using SVD, which was performed as described in another previous study [71], were used in order to estimate the muscle stiffness and viscous characteristics under low power output [74]. The authors then observed a progressive increase in muscle stiffness as the pedal rate also increased. The stiffness was approximated at $P<0.025$, with a decision coefficient of 0.984 . However, the viscous coefficient was determined to not increase. A possible reason for these findings is the rubbing between active and inactive muscle fibers due to the force exerted in direct proportion to the velocity. Clearly, this study has examined the muscle activity using EMG and found that $\mathrm{EMG}_{\mathrm{RMS}}$ showed changes at different pedaling rates in various subjects. In contrast, similar mean $\mathrm{EMG}_{\mathrm{RMS}}$ values were found at 40,60 , and $80 \mathrm{rpm}$. Thus, future studies should elucidate the mechanism underlying the associations among muscle stiffness, viscous coefficients, and muscle function.

More recently, another study [73] has examined the stiffness obtained based on the GM dependency on gait speed. During treadmill training, stimulated and nonstimulated MMGs were recorded from the GM, and the evoked MMG signals were then used to identify the stiffness index based on the corresponding natural frequencies. The researchers reported that the frequencies and thus the stiffness increased as the gait speed increases.

The authors of a previous study [59] have also used changes in the joint angle torque to estimate stiffness. The responses from electrical excitation of the GM, namely, the EMG, MMG, and torque, were recorded to estimate the muscle stiffness before and after static stretching. After dividing the information into electrochemical and mechanical components, the offline $\mathrm{R}$ - Delay ${ }_{\text {TOT }}$ was determined based on the force, EMG, and MMG signals recorded at $P^{\mathrm{T}}$. A decrease in the main electromechanical component was then observed as the joint angle increased before and after static stretching. This study has also determined that during muscle relaxation, an increased dorsiflexion angle could be attributed to joint stiffness rather than the electrochemical process.

Authors of this study have further demonstrated that the anthropometric features and geometry of the limbs can contribute to joint stiffness and found that the frequency 
components increased as stiffness increases [76], even though the so-called viscous coefficient did not seem to vary. In addition, the changes in the joint angle torque after stretching tasks have exhibited a progressive decrease in electromechanical delay components with increases in the joint angle at pT [72]. As what has been discussed in a previous study [77], the effects of inactive and active muscles on the viscous coefficient [75], subcutaneous tissue [73], MTU stiffness, and joint angles should be considered in further studies [78]. It can be concluded that the anthropometric, electrochemical, and mechanical components, daily physical tasks, and geometry of the muscles are of importance in terms of estimating for muscle stiffness using myographic signals.

\section{Torque assessment}

As presented in Table 9, nine studies [79-87] have reported that torque is mirrored by the MMG features from electrically contracted muscles. The authors of one of these previous studies [79] used a combination of MMG and torque signals in determining the dependency of the contractile properties of a muscle on its length. The BB was electrically stimulated using $10-$ and $30-\mathrm{Hz}$ fused and unfused tetanic stimulation, and then, evoked flexion torque was measured at six joint angles ranging from $75^{\circ}$ to $150^{\circ}$ at $15^{\circ}$ increments. The RMS values of both the torque and MMG signals obtained with different stimulation frequencies confirmed the reliability of MMG for evaluating the contractile properties of muscles. In addition, the findings have demonstrated that the torque fluctuates with variations in the muscle length (Table 10).

Some researchers [87] have examined the effects of aging on muscles through a comparison of MMG and EMG features with torque's profiles. The muscles of old and young individuals were subjected to electromyostimulation before and after $10 \mathrm{~s}$ and then less than $100 \% \mathrm{MVC}$, wherein both MMG and $P^{\mathrm{T}}$ were observed to have been significantly increased, but the $\mathrm{M}$-waves remained steady. However, no effect of aging was detected. During voluntary contraction, the MMG and EMG profiles in young and old men exhibited similar shapes, which indicated that the electrical and mechanical properties of voluntary or artificial stimulation of muscles exhibited different behaviors at different ages.

Many studies have used a combination of MMG and torque signals in an effort to characterize muscle mechanics. Using short and long stimulation pulses, the detected MMG and torque signals yielded two transfer functions, and these functions were analyzed to characterize the TA muscle joints. After stimulation intensity was delivered, the torque was determined offline based on the tension recorded from the foot and ankle lever arm. Therefore, the in vivo mechanical properties of muscle joints obtained using short stimulation protocols were obtained from MMG and torque signals [80]. An analysis of the torque, MMG, and EMG signals obtained under NMES with triangular trains with varying frequencies and amplitudes has yielded the muscle input/output relationship of the TA under static contraction and thus showed the module of muscle force production [81]. The relationship between the stimulation frequencies yielded the average torque, MMG, and EMG signals for each 5\% of the $\Delta$ frequency range from $2 \mathrm{~Hz}(0 \%)$ to $35 \mathrm{~Hz}$ $(100 \%)$. As per experimental results, an additional torque corresponds to an increase in the extra-muscle displacement (MMG) during down-going ramp (DGR) rather than upgoing ramp (UGR) for both frequency and amplitude triangles. In addition, the authors 


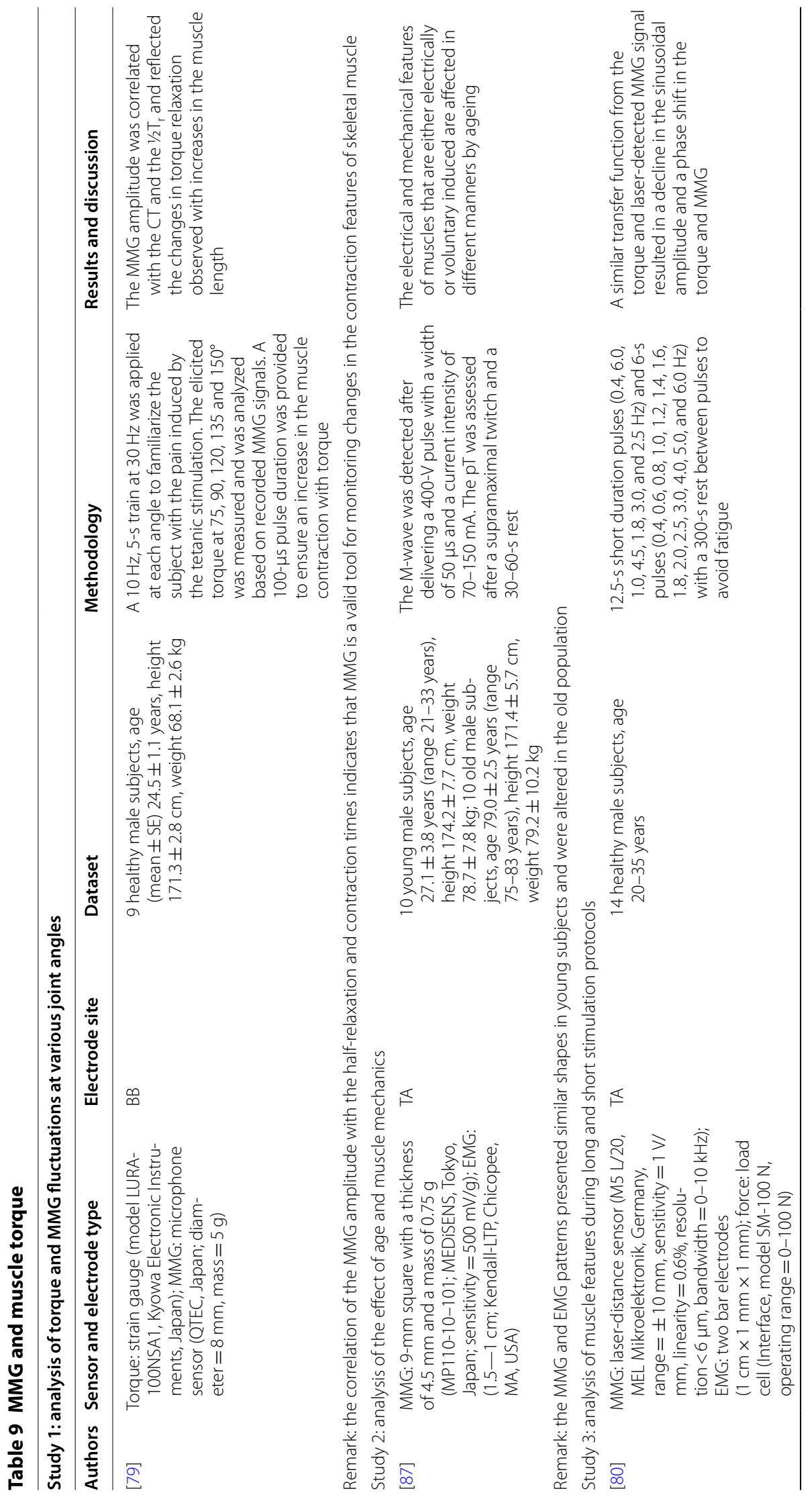




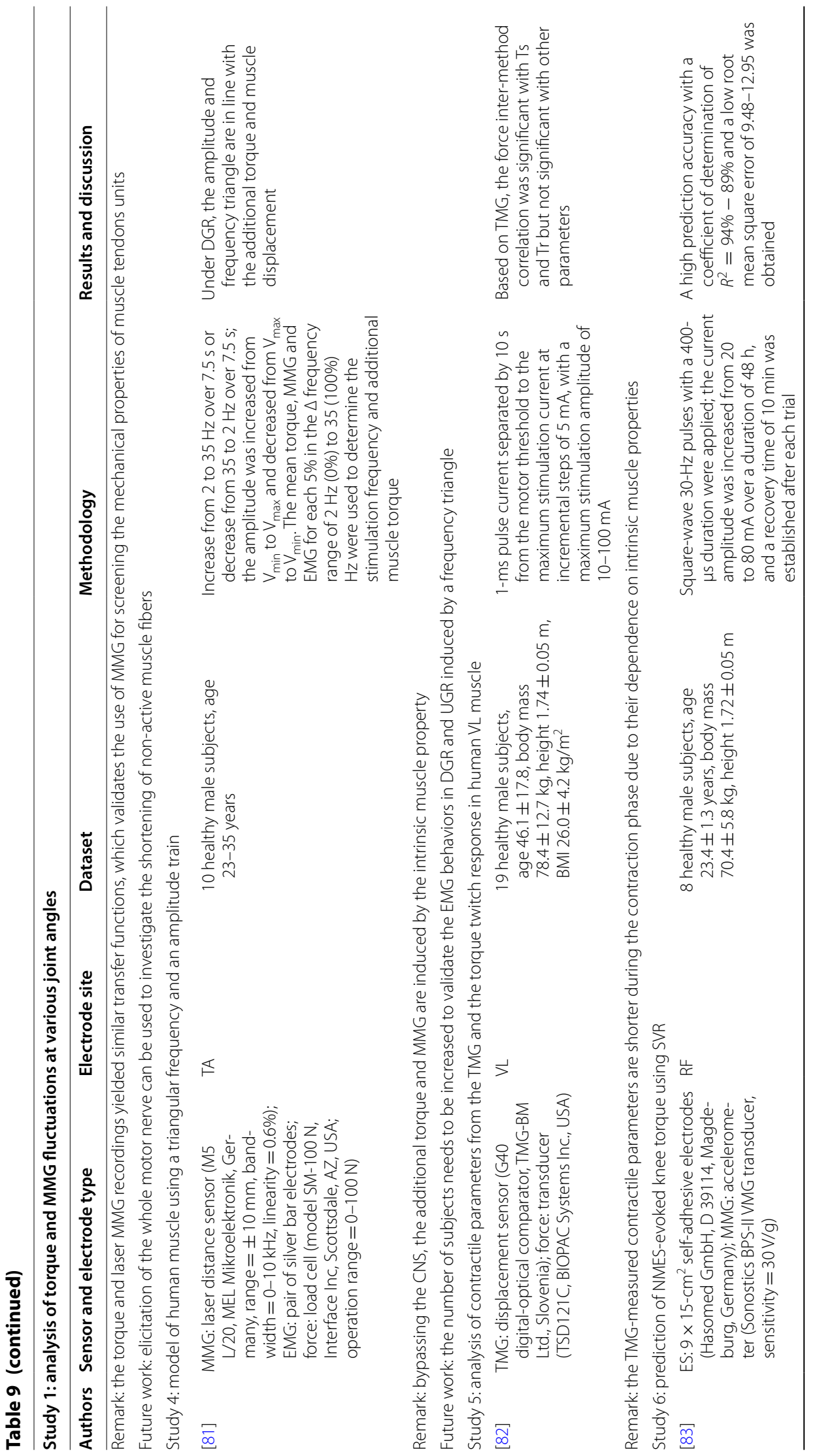




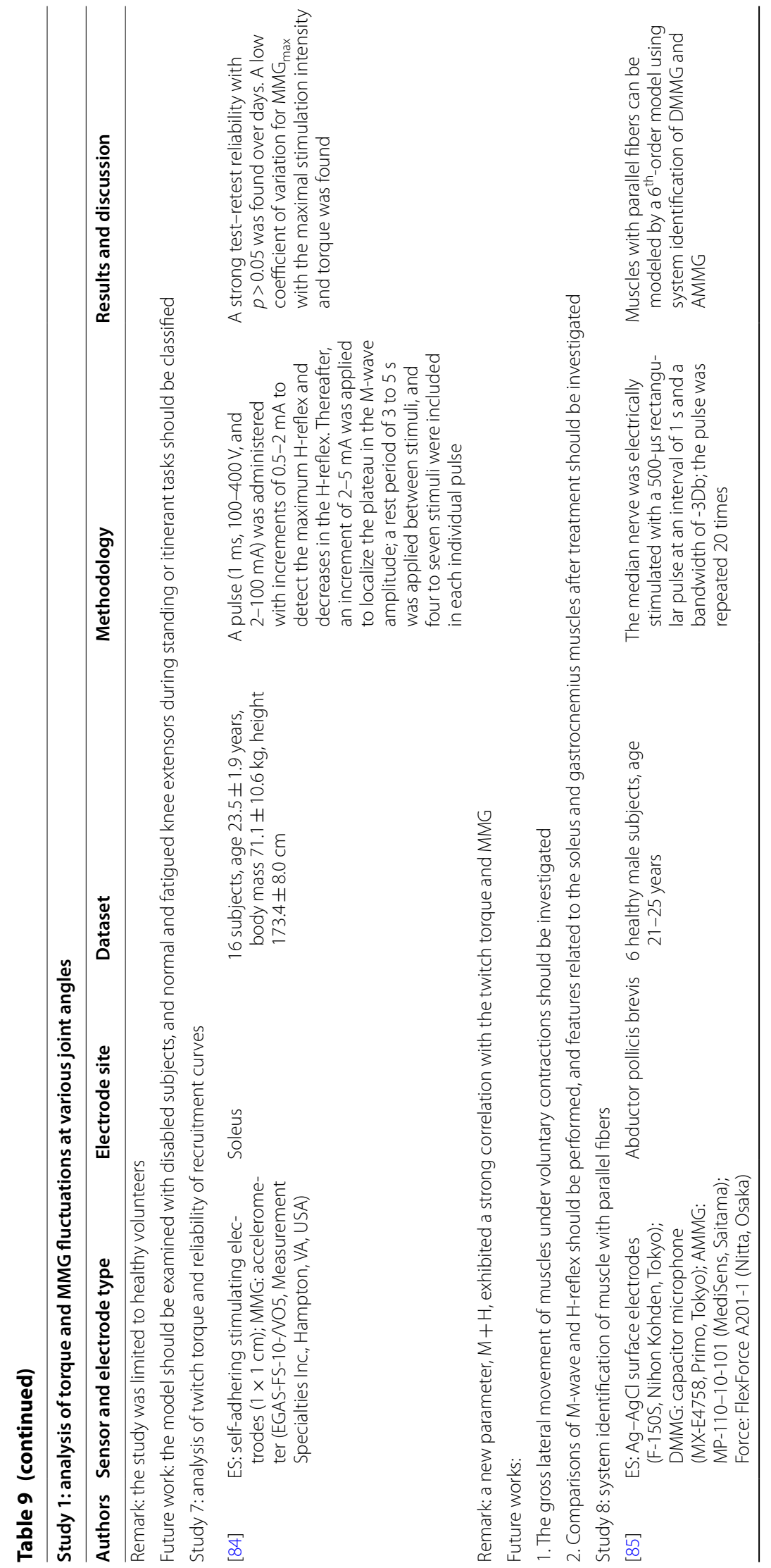




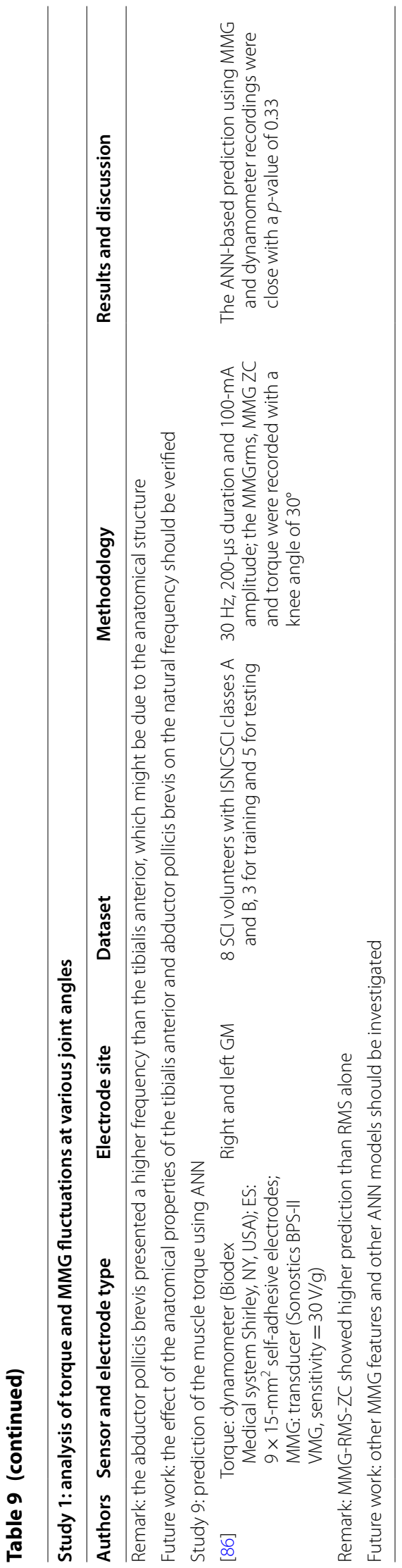


suggest that stimulation parameters close to physiological quantities indicate the influence of intrinsic muscle factors on additional torque and MMG signals.

However, the findings obtained in a previous study [82] have been deemed controversial. The longitudinal torque and transverse TMG have yielded differences in the contractile parameters of the VL. Therefore, these findings indicate that the TMG mechanical responses can reflect the intrinsic contractile parameters of skeletal muscles.

A study conducted a year later [83] used computational intelligence to solve the complex mathematical computation derived in the above-mentioned previous studies [82]. UsingMMG ${ }_{p-p}, M_{M} G_{\mathrm{RMS}}$, the knee angle, and the levels of NMES parameters, the SVR was trained to predict torque; then, the knee torque was in line with the dynamometer readings. The high correlation and reduced root mean square error only confirmed the efficiency of the developed technique in terms of estimating the knee torque and the accuracy of the laboratory results. Similarly, the variability in the neurological function of SCI patients determined in this study [83] was addressed using artificial intelligence, which was performed in a subsequent study [86]. After delivering electrical impulses to the quadriceps muscle groups of three SCI subjects, the knee torque estimated using a dynamometer was significantly correlated with the ANN-predicted torque. Therefore, the authors highlight the usefulness of ANN for real-time torque estimation.

Thereafter, the methods were further validated in a later study [84]. Using increasing stimulation amplitudes, the peak-to-peak torque was obtained from an average of the 20 highest data points, and the M-wave, H-reflex, and MMG responses were also determined using the peak-to-peak amplitudes. Thus, the combination of M-waves and $\mathrm{H}$-reflexes was proposed for estimation of the plantar flexion torque using Cybex 6000. The study then introduced the $\mathrm{M}+\mathrm{H}$ parameter, which was strongly correlated with the twitch torque and MMG. Hence, the study was able to determine that MMG was not affected by stimulus artifacts and is thus recommended for estimating the maximal twitch torque. This pioneering work on NMES and mathematical models continues to guide research in the characterization of muscles, particularly small muscles. Using SVD, the transfer function between torque and MMG reported by a previous study [85] showed that the activity of small, thin, and parallel muscle fibers can be mathematically modeled through a combination of DMMG and AMMG. The findings of these studies have supported the use of muscle mechanics, anthropometry, and age difference in terms of characterizing the muscle torque. Machine learning $[83,86]$ was also determined to provide greater results which support the hypothesis that sophisticated signal processing may provide promising future improvements in terms of MMG-based muscles studies.

\section{Miscellaneous/clinical monitoring}

MMG can reportedly be used in real-time clinical monitoring [88-93]. The authors of a related study [92] were able to detect an MMG response of the target nerve to a specific stimulation current to verify the rate of nerve root decompression, wherein a decrease in the stimulation threshold of $\geq 1 \mathrm{~mA}$ was observed in $98 \%$ of the test subjects. The affected nerve root maintained a stimulus threshold of $1 \mathrm{~mA}$ following decompression but exhibited an increase in the amplitude of the MMG signal response. The technique can thus adequately guide a surgeon in cases in which decompression is deemed 


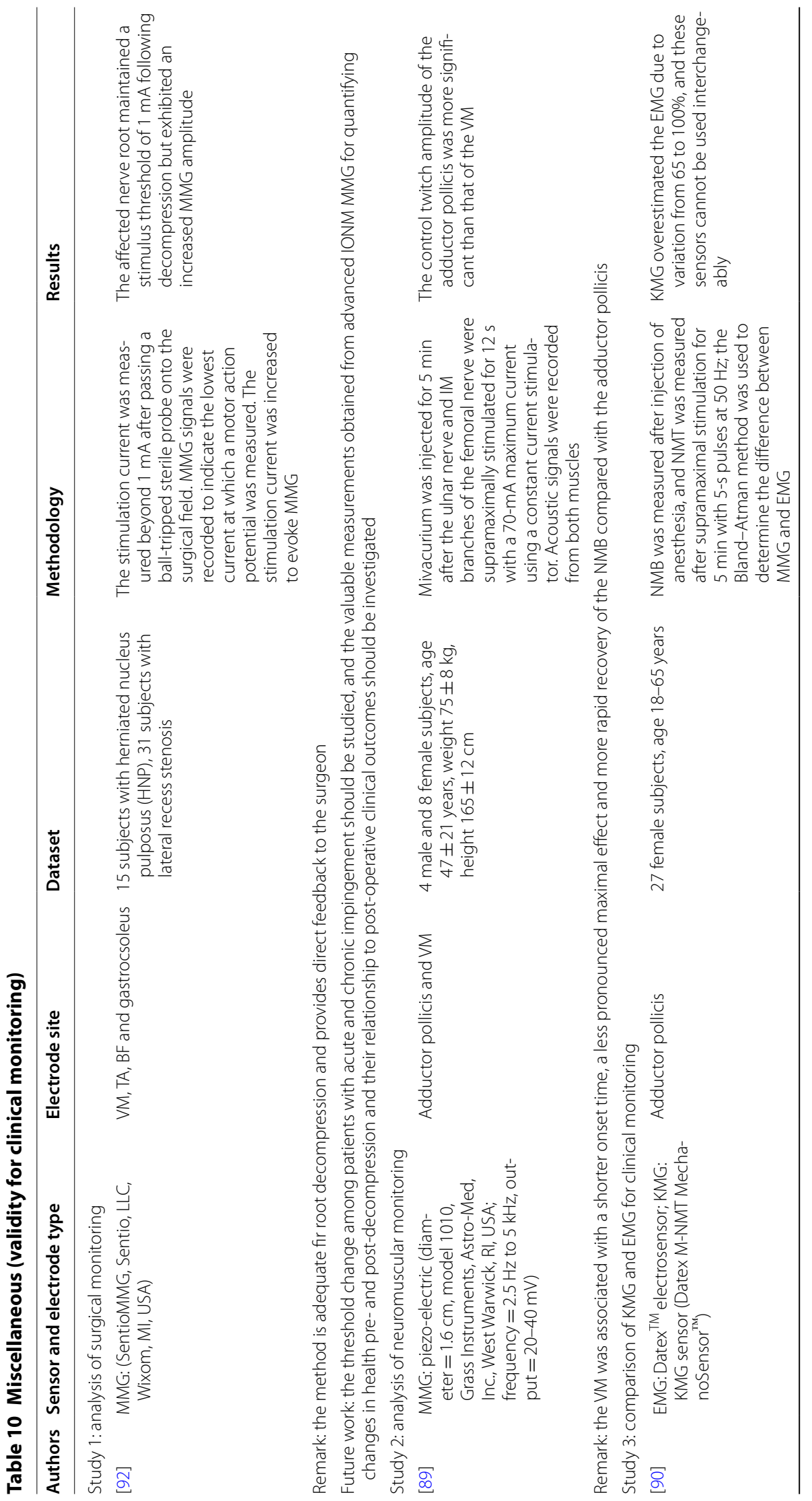




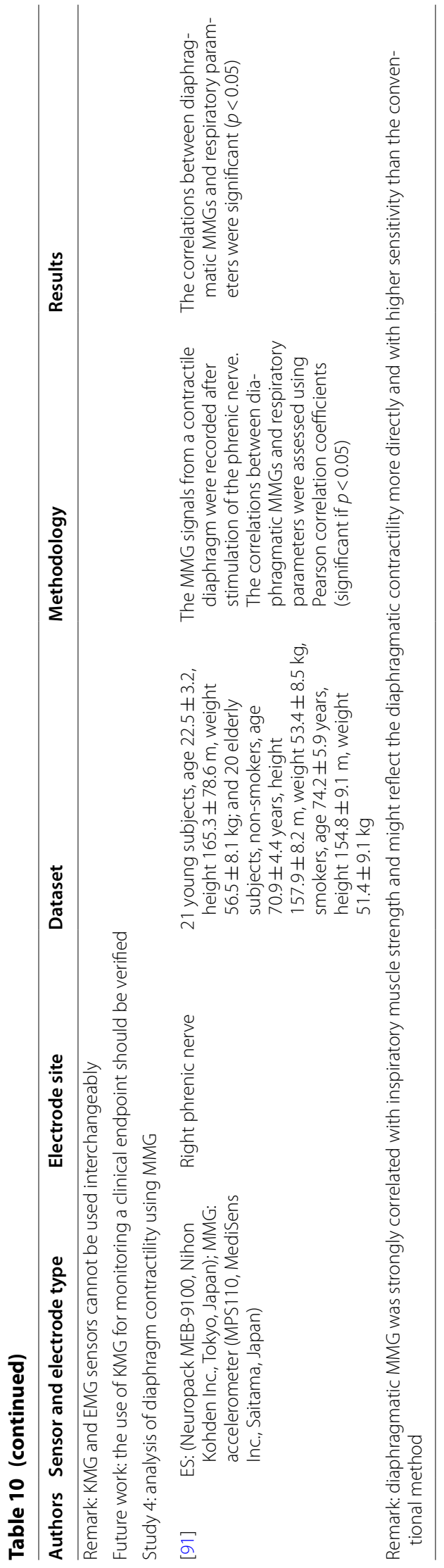


uncertain. Furthermore, two other studies [88, 89] verified the validity of KMG and PMG in neuromuscular monitoring. Prior to general anesthesia, the ulnar nerve has received supramaximal train-of-four stimulation for every $12 \mathrm{~s}$ at $0-70 \mathrm{~mA}$. Based on the concordance correlation coefficient, the recovery of NMB determined using KMG and PMG agreed with that obtained based on the MMG response. In contrast in [86], the features of KMG appear to exhibit a high TOF ratio in NMB monitoring. Some researchers have compared the KMG data recorded using a Datex M-NMT MechanoSensor with EMG data recorded with a Datex ElectroSensor and found a higher recovery in terms of TOF $\%$ with KMG compared with EMG. However, the researchers stated that the $90 \%$ recovery rate of KMG makes it less preferable for NMB than EMG. Thus, further validation of these findings in clinical settings is needed [90].

In another study [93], the BB was synchronously stimulated with a programmable function generator providing a 1-ms pulse, and 3D images were acquired through ultrafast acquisition in the transverse plane at each position. The Tc and $1 / 2 \mathrm{Tr}$ matched the in vitro and in vivo surface MMG responses. In addition, the muscle fiber bundle was retrieved and agreed with the echogenic architecture of the muscle. Further, the researchers argued that the technique can be used to remedy the inability of diffusion tensor imaging in providing the mechanical function of a muscle, allowing for easy recruitment of muscle fibers. Therefore, the authors encourage further verification of the validity of myography-based screening of the functions of other muscles.

Several of the available techniques for respiratory assessment have been determined to involve maximal inspiratory and expiratory mouth pressures. After electrical excitation of the phrenic nerve, the MMG responses from the contracted diaphragm correlated with the respiratory muscle strength and provided higher sensitivity than the conventional method [91]. Altogether, the techniques of MMG, KMG, and PMG through NMES are presented as interesting tools to evaluate muscle behaviors in clinical setting with no physical efforts. Taking advantage of the myographic signals from NMES to monitor specific muscle status, more research is needed to verify the functions of other muscles.

\section{Conclusion}

The studies examined in this review presented a comprehensive overview of approaches used for assessing muscle activities based on MMG through electrical activation protocols. The technique has been used to identify the contractile properties of muscles in specific tasks to denote the changes that take place after electrical activation for recovery and function monitoring. Our findings support the hypothesis that MMG coupled with NMES is suitable for muscle function assessment. However, like EMG, the MMG signal alone is prone to crosstalk contamination from nearby muscles and those beneath the skin. Coupled with electro-muscle stimulation, unlike EMG, the MMG signal showed no interference from electrical signals. Thus, taking advantage of electrical excitation, the examination of the activity of a single muscle from a group of muscles could be a tool to quantify the crosstalk. In this review, it was also shown that a combination of MMG, NMES, and task training provided robust outcomes. Hence, disabled people can be psychologically motivated, thus familiarizing with electric sensation in functional training. Nevertheless, the application of NMES differs depending on one's susceptibility 
to intensities of stimulation and the degree of disability when applied for rehabilitation purposes. It will be important to establish if MMG and electrical muscle stimulation can yield a functional rehabilitation program for people with or without complete SCI. Studies have shown that MMG parameters obtained through electrical muscle stimulation protocols have reliable physiological parameters of targeted muscles that correlate with torque and have been also used for fatigue, stiffness, and endurance assessments of skeletal muscles using mathematical computations. Our findings from the statistical analysis of MMG and electromyostimulation coupling suggest the use of artificial intelligence as a potential new approach.

\begin{abstract}
Abbreviations
Delay Tот: $_{\text {: }}$ Delay in force development; $1 / 2 T_{\mathrm{r}}$ : Half relaxation time; Ag-PDMS: Silver-polydimethylsiloxane; AMMG: Acceleration mechanomyography; ANN: Artificial neural network; CDS: Contact displacement sensor; DGR: Down-going ramp; $D_{m}$ : Maximum displacement; DMMG: Displacement mechanomyography; EMG: Electromyography; FRDA: Friedreich ataxia; GLM: Gloss lateral movement; GM: Gastrocnemius; ICC: Intraclass correlation coefficient; IED: Inter-electrode distance; KMG: Kinemyography; LCE: Leg cycling exercise; LDS: Laser displacement sensor; LFF: Low-frequency fatigue; MCS: Muscle contraction sensor; MEMS: Microelectromechanical system; MF: Mean frequency; MFCC: Mel frequency cepstral coefficient; MMG: Mechanomyography; MPF: Mean power frequency; MTU: Muscle-tendon unit; MU: Motor unit; MVC: Maximum voluntary contraction; MVIC: Maximum voluntary isometric contraction; NIRS: Near-infrared spectroscopy; NMES: Neuromuscular electrical stimulation; PAP: Post activation potentiation; $\mathrm{pT}$ : Peak torque; RF: Rectus femoris; RMS: Root mean square; SCI: Spinal cord injury; SEMG: Surface electromyography; SOL: Soleus; SVD: Singular value decomposition; SVM: Support vector machine; SVR: Support vector regression; TB: Triceps brachii; TMG: Tensiomyography; UGR: Up-going ramp; VL: Vastus lateralis; VM: Vastus medialis; VMG: Vibromyography; VML: Vastus medialis longus; VMO: Vastus medialis obliquus.
\end{abstract}

Acknowledgements

This research was funded by the University of Rwanda, the Regional Centre of Excellence for Biomedical Engineering and E-Health (UR-CEBE), and the research platform was provided by the Centre for telecommunication, Research and Innovation, Universiti Teknikal Malaysia Melaka (CeTRI-UTeM).

\title{
Authors' contributions
}

UR conceptualized the research methodology and original drafting. KS supervised and validated the research framework. IDS edited and proofread the paper. All authors read and approved the final manuscript.

\section{Funding}

Not applicable.

Availability of data and materials

Not applicable.

Ethical approval and consent to participate

Not applicable.

Consent to participate

Not applicable.

Consent for publication

Not applicable.

Competing interests

The authors declare that they have no competing financial interest that influenced the research work reported in this article.

\section{Author details}

${ }^{1}$ Fakulti Kejuruteraan Elektronik \& Kejuruteraan Komputer, Universiti Teknikal Malaysia Melaka, Tunggal, Malaysia.

2 Regional Centre of Excellence in Biomedical Engineering and E-Health, University of Rwanda, PO BOX 4285, Kigali,

Rwanda. ${ }^{3}$ Pusat Bahasa \& Pembangunan Insan, Universiti Teknikal Malaysia Melaka, Tunggal, Malaysia.

Received: 13 April 2020 Accepted: 11 December 2020

Published online: 03 January 2021

\section{References}

1. Zhang Q, Hosoda R, Venture G. Human joint motion estimation for electromyography (EMG)-based dynamic motion control. In IEEE International Conference Engineering in Medicine \& Biology Society, Osaka, Japan. 2013. p. 21-24 
2. Kuriki HU, De Azevedo FM, Takahashi LS, Mello EM, de Faria Negrão Filho R, Alves N. The relationship between electromyography and muscle force. In EMG methods evaluation muscle and nerve function, M. Schwartz, E.D. Rijeka: Interchopen. 2012. p. 31-54.

3. Phinyomark A, Khushaba RN, Scheme E. Feature extraction and selection for myoelectric control based on wearable EMG sensors. Sensors. 2018;18(5):1-17.

4. Phinyomark A, Quaine F, Charbonnier S, Serviere C, Tarpin-Bernard F, Laurillau Y. EMG feature evaluation for improving myoelectric pattern recognition robustness. Expert Syst Appl. 2013;40(12):4832-40.

5. Wang D, Wu H, Xie C, Gao L. Suppression of motion artifacts in multichannel mechanomyography using multivariate empirical mode decomposition. IEEE Sens J. 2019;19(14):5732-9.

6. Divakaran S, Abraham S, Janney JB, Umashaukar G. Analyzing EMG and MMG signals for MMG driven bionic arm Analyzing EMG and MMG signals for MMG driven bionic arm. J Chem Pharm Res. 2015;7(4):56-61.

7. Cè E, Rampichini S, Esposito F. Novel insights into skeletal muscle function by mechanomyography: from the laboratory to the field. Sport Sci Health. 2015;11(1):1-28.

8. Talib I, Sundaraj K, Lam CK, Jawad H, Asraf Ali M. A review on crosstalk in myographic signals. Eur J Appl Physiol. 2018;119(1):9-28.

9. Talib I, Sundaraj K, Lam CK, Nabi FG. Development of a standalone application to measure crosstalk in MMG signals from forearm muscles during wrist postures. J Telecommun Electron Comput Eng. 2018;10(2):103-6.

10. Antonelli MG, Beomonte Zobel P, Giacomin J. Use of MMG signals for the control of powered orthotic devices: development of a rectus femoris measurement protocol. Assist Technol. 2009;21(1):1-12.

11. Madeleine P, Cescon C, Farina D. Spatial and force dependency of mechanomyographic signal features. J Neurosci Methods. 2006;158(1):89-99.

12. Anders JP, Smith CM, Keller JL, et al. Inter- and intra-individual differences in EMG and MMG during maximal, bilateral, dynamic leg extensions. Sports. 2019;7(7):175.

13. Fara S, Gavriel C, Vikram CS, Faisal AA. Prediction of arm end-point force using multi-channel MMG. In IEEE Int. Conf. Wear Impl. Body Sen. Netw., Washngton, DC. 2014. p. 27-32.

14. Ibitoye MO, Hamzaid NA, Hasnan N, et al. Torque and mechanomyogram relationships during electrically-evoked isometric quadriceps contractions in persons with spinal cord injury. Med Eng Phys. 2016;38(8):767-75.

15. Silva J, Heim W, Chau T. A self-contained mechanomyography-driven externally powered prosthesis. Arch Phys Med Rehabil. 2005:86(10):2066-70.

16. Marusiak J, Jaskólska A, Jarocka E, Najwer W, Kisiel-Sajewicz K, Jaskólski A. Electromyography and mechanomyography of elbow agonists and antagonists in Parkinson disease. Muscle Nerve. 2009;40(2):240-8.

17. Beck TW, Housh TJ, Johnson GO, et al. Does the frequency content of the surface mechanomyographic signal reflect motor unit firing rates? A brief review. J Electromyogr Kinesiol. 2007;17(1):1-13.

18. Beck TW, Housh T, Fry AC, Cramer JT, Weir J, Schilling B, Falvo M, Moore C. MMG-EMG cross spectrum and muscle fiber type. Int J Sports Med. 2009;30(7):538-44.

19. Orizio C, Gobbo M, Diemont B, Esposito F, Veicsteinas A. The surface mechanomyogram as a tool to describe the influence of fatigue on biceps brachii motor unit activation strategy. Historical basis and novel evidence. Eur J Appl Physiol. 2006;90(3-4):26-36.

20. Jones S, Man WD, Gao W, Higginson IJ, Wilcock A, Maddocks M. Neuromuscular electrical stimulation for muscle weakness in adults with advanced disease. Cochrane Database Syst Rev. 2016;10:1-66.

21. Vromans M, Faghri P. Electrical stimulation frequency and characteristics: effects on force and fatigue skeletal muscle. Eur J Transl Myol. 2017;27(4):239-45.

22. Faller L, Nogueira Neto GN, Button VL, Nohama P. Muscle fatigue assessment by mechanomyography during application of NMES protocol. Brazilian J Phys Ther. 2009;13(5):422-9.

23. Busk H, Stausholm MB, Lykke L, Wienecke T. Electrical stimulation in lower limb during exercise to improve gait speed and functional motor ability 6 months poststroke. Review with meta-analysis. J Stroke Cerebrovasc Dis. 2019;29(3):104565.

24. Megía García A, Serrano-Muñoz D, Taylor J, Avendaño-Coy J, Gómez-Soriano J. Transcutaneous spinal cord stimulation and motor rehabilitation in spinal cord injury: a systematic review. Neurorehabil Neural Repair. 2019;34(1):3-12.

25. Cooper MA, Herda TJ, Vardiman JP, Gallagher PM, Fry AC. Relationships between skinfold thickness and electromyographic and mechanomyographic amplitude recorded during voluntary and non-voluntary muscle actions. J Electromyogr Kinesiol. 2014;24(2):207-13.

26. Tous-Fajardo J, Moras G, Rodríguez-Jiménez S, Usach R, Doutres DM, Maffiuletti NA. Inter-rater reliability of muscle contractile property measurements using non-invasive tensiomyography. J Electromyogr Kinesiol. 2010;20(4):761-6.

27. Šimunič B, Koren $\mathrm{K}$, Rittweger J, et al. Tensiomyography detects early hallmarks of bed-rest-induced atrophy before changes in muscle architecture. J Appl Physiol. 2019;126(4):815-22.

28. Travnik L, Djordjevič S, Rozman S, Hribernik M, Dahmane R. Muscles within muscles: a tensiomyographic and histochemical analysis of the normal human vastus medialis longus and vastus medialis obliquus muscles. J Anat. 2013;222(6):580-7.

29. McAndrew DJ, Rosser NA, Brown JM. Mechanomyographic measures of muscle contractile properties are influenced by the duration of the stimulatory pulse. J Appl Res Clin Exp Ther. 2006;6(1):142.

30. Ohta Y. The relationship between changes in joint kinematics parameters and mechanomyographic signals during non-isometric contraction in human skeletal muscle. Acta Bioeng Biomech. 2013;15(2):97-104.

31. Latella C, Ruas CV, Mesquita RN, Nosaka K, Taylor JL. Test-retest reliability of elbow flexor contraction characteristics with tensiomyography for different elbow joint angles. J Electromyogr Kinesiol. 2019;45:26-32.

32. Papcke C, Krueger E, Olandoski M, Nogueira-Neto GN, Nohama P, Scheeren EM. Investigation of the relationship between electrical stimulation frequency and muscle frequency response under submaximal contractions. Artif Organs. 2019;42(6):655-63.

33. Miyamoto N, Mitsukawa N, Sugisaki N, Fukunaga T, Kawakami Y. Joint angle dependence of intermuscle difference in postactivation potentiation. Muscle Nerve. 2010;41(4):519-23. 
34. Shima N, Rice CL, Ota Y, Yabe K. The effect of postactivation potentiation on the mechanomyogram. Eur J Appl Physiol. 2006;96(1):17-23.

35. Toca-Herrera JL, Gallach JE, Gómis M, González LM. Cross-education after one session of unilateral surface electrical stimulation of the rectus femoris. J Strength Cond Res. 2008;22(2):614-8.

36. Mazzinari G, Errando CL, Díaz-Cambronero O, Martin-Flores M. Influence of tetanic stimulation on the staircase phenomenon and the acceleromyographic time-course of neuromuscular block: a randomized controlled trial. J Clin Monit Comput. 2018;33(2):325-32.

37. Armstrong WJ. Wavelet - based intensity analysis of the mechanomyograph and electromyograph during the $\mathrm{H}$-reflex. Eur J Appl Physiol. 2014;114(12):2571-8.

38. Ibrahim SN, Rahman FA, Rosli S. Characterization of screen printed Ag-PDMS flexible electrode for electrical characterization of screen printed Ag-PDMS flexible electrode for electrical muscle stimulation (EMS). Indones J Electr Eng Informatics. 2017:5(4):295-303.

39. Rafolt D, Gallasch E. Surface myomechanical responses recorded on a scanner galvanometer. Med Biol Eng Comput. 2002;40(5):594-9.

40. Žagar T, Križaj D. Validation of an accelerometer for determination of muscle belly radial displacement. Med Biol Eng Comput. 2005:43(1):78-84.

41. Uchiyama T, Shinohara K. Comparison of displacement and acceleration transducers for the characterization of mechanics of muscle and subcutaneous tissues by system identification of a mechanomyogram. Med Biol Eng Comput. 2013;51(1-2):165-73.

42. Seidl L, Tosovic D, Brown JM. Test-retest reliability and reproducibility of laser-vs contact—displacement sensors in mechanomyography: implications for musculoskeletal research. J Appl Biomech. 2017;33(2):130-6.

43. Mohamad NZ, Hamzaid NA, Davis GM, Wahab A, Khairi A, Hasnan N. Mechanomyography and torque during FESevoked muscle contractions to fatigue in individuals with spinal cord injury. Sensors. 2017;17(7):1-15.

44. Ng MY, Pourmajidian M, Hamzaid NA. Mechanomyography sensors for detection of muscle activities and fatigue during Fes-evoked contraction. In IEEE International Functional Electrical Stimulation Society, Kuala Lumpur; 2014. p. 1-3.

45. Islam MA, Sundaraj K, Ahmad RB, Ahamed NU, Ali MA. Mechanomyography sensor development, related signal processing, and applications: a systematic review. IEEE Sens J. 2013;13(7):2499-516.

46. Talib I, Sundaraj K, Lam CK. Choice of mechanomyography sensors for diverse types of muscle activities. J Telecommun Electron Comput Eng. 2018;10(1-13):79-82.

47. Blangsted AK, Sjøgaard G, Madeleine P, Olsen HB, Søgaard K. Voluntary low-force contraction elicits prolonged low-frequency fatigue and changes in surface electromyography and mechanomyography. J Electromyogr Kinesiol. 2005;15(2):138-48.

48. Adamo DE, Martin BJ, Johnson PW. Vibration-induced muscle fatigue, a possible contribution to musculoskeletal injury. Eur J Appl Physiol. 2002;88(1-2):134-40.

49. Cè E, Rampichini S, Monti E, Venturelli M, Limonta E, Esposito F. Changes in the electromechanical delay components during a fatiguing stimulation in human skeletal muscle: EMG, MMG and force combined approach. Eur J Appl Physiol. 2016:117(1):95-107.

50. Rampichini S, Cè E, Limonta E, Esposito F. Effects of fatigue on the electromechanical delay components in gastrocnemius medialis muscle. Eur J Appl Physiol. 2013;114(3):639-51.

51. Cè E, Rampichini S, Limonta E, Esposito F. Fatigue effects on the electromechanical delay components during the relaxation phase after isometric contraction. Acta Physiol. 2014;211(1):82-96.

52. Cè E, Rampichini S, Limonta E, Esposito F. Torque and mechanomyogram correlations during muscle relaxation: effects of fatigue and time-course of recovery. J Electromyogr Kinesiol. 2013;23(6):1295-303.

53. Macgregor LJ, Ditroilo M, Smith IJ, Fairweather MM, Hunter AM. Reduced radial displacement of the gastrocnemius medialis muscle after electrically elicited fatigue. J Sport Rehabil. 2016;25(3):241-7.

54. Naeem J, Hamzaid NA, Illam MA, Azman AW, Bijak M. Mechanomyography-based muscle fatigue detection during electrically elicited cycling in patients with spinal cord injury. Med Biol Eng Comput. 2019;56(6):1199-211.

55. Islam MA, Hamzaid NA, Ibitoye MO, Hasnan N, Wahab AK, Davis GM. Mechanomyography responses characterize altered muscle function during electrical stimulation-evoked cycling in individuals with spinal cord injury. Clin Biomech. 2018;58:21-7.

56. Jo M, Ahn S, Kim J, Koo B, Jeong Y, Kim S, Kim Y. Mechanomyography for the measurement of muscle fatigue caused by repeated functional electrical stimulation. Int J Precis Eng Manuf. 2018;19(9):1405-10.

57. Gobbo M, Cè E, Diemont B, Esposito F, Orizio C. Torque and surface mechanomyogram parallel reduction during fatiguing stimulation in human muscles. Eur J Appl Physiol. 2006;97(1):9-15.

58. Krueger E, Scheeren EM, Nogueira-Neto GN, Button VL, Nohama P. Correlation between spectral and temporal mechanomyography features during functional electrical stimulation. Res Biomed Eng. 2016;32(1):85-91.

59. Esposito F, Cè E, Rampichini S, Veicsteinas A. Acute passive stretching in a previously fatigued muscle: electrical and mechanical response during tetanic stimulation. J Sports Sci. 2009;27(12):1347-57.

60. Ohta Y, Shima N, Yabe K. In vivo behaviour of human muscle architecture and mechanomyographic response using the interpolated twitch technique. J Electromyogr Kinesiol. 2009;19(3):154-61.

61. Cè E, Paracchino E, Esposito F. Electrical and mechanical response of skeletal muscle to electrical stimulation after acute passive stretching in humans: a combined electromyographic and mechanomyographic approach. J Sport Sci. 2008;26(14):1567-77.

62. Willingham TB, Melbourn J, Moldavskiy M, McCully KK, Backus D. Effect of antigravity treadmill training on muscle oxidative capacity, muscle endurance, and walking function in a person with multiple sclerosis. Int J MS Care. 2018:20(4):186-90

63. McCully KK, Moraes C, Patel SV, Green M, Willingham TB. Muscle-specific endurance of the lower back erectors using electrical twitch mechanomyography. J Funct Morphol Kinesiol. 2019;4(1):12.

64. Decker MJ, Griffin L, Abraham LD, Brandt L. Alternating stimulation of synergistic muscles during functional electrical stimulation cycling improves endurance in persons with spinal cord injury. J Electromyogr Kinesiol. 2010;20(6):1163-9. 
65. Bossie HM, Willingham TB, Schoick RA, O'Connor PJ, McCully KK. Mitochondrial capacity, muscle endurance, and low energy in Friedreich ataxia. Muscle Nerve. 2017;56(4):773-9.

66. Norali AN, Abdullah AH, Zakaria Z, Rahim NA, Nataraj SK. Human breathing classification using electromyography signal with features based on mel-frequency cepstral coefficients. Int J Integr Eng. 2017;9(4):85-92.

67. Esposito F, Limonta E, Cè E. Passive stretching effects on electromechanical delay and time course of recovery in human skeletal muscle: new insights from an electromyographic and mechanomyographic combined approach. Eur J Appl Physiol. 2010;11(3):485-95.

68. Esposito F, Limonta E, Cè E. Time course of stretching-induced changes in mechanomyogram and force characteristics. J Electromyogr Kinesiol. 2011;21(5):795-802.

69. Kimura T, Hamada T, Ueno LM, Moritani T. Changes in contractile properties and neuromuscular propagation evaluated by simultaneous mechanomyogram and electromyogram during experimentally induced hypothermia. J Electromyogr Kinesiol. 2003;13(5):433-40.

70. Yung M, Mathiassen SE, Wells RP. Variation of force amplitude and its effects on local fatigue variation of force amplitude and its effects on local fatigue. Eur J Appl Physiol. 2012;112(11):3865-79.

71. Uchiyama T, Tomoshige T. System identification of velocity mechanomyogram measured with a capacitor microphone for muscle stiffness estimation. J Electromyogr Kinesiol. 2017;3:57-637.

72. Longo S, Devoto M, Monti E, et al. Acute effects of static stretching on skeletal muscle relaxation at different ankle joint angles. Sport Sci Health. 2016;12(3):429-36.

73. Matsue Y, Uchiyama T. Medial gastrocnemius muscle stiffness dependent on gait speed. In: Proceedings on Transactions of Japanese Society for Medical and Biological Engineering. 2017; 55:534-535.

74. Uchiyama T, Saito K. Stiffness and viscosity of the vastus lateralis muscle in cycling exercises at low constant power output stiffness and viscosity of the vastus lateralis muscle in cycling exercises at low constant power output. Adv Biomed Eng. 2018;7:124-30.

75. Uchiyama T, Saito K, Shinjo K. Muscle stiffness estimation using a system identification technique applied to evoked mechanomyogram during cycling exercise. J Electromyogr Kinesiol. 2015;25(6):847-52.

76. Fukawa T, Uchiyama T. System identification of evoked mechanomyogram to clarify lower limb muscle stiffness in treadmill walking. Trans Japanese Soc Med Biol Eng. 2016;52:1-6.

77. Jarocka E, Marusiak J, Kumorek M, Jaskólska A, Jaskólski A. Muscle stiffness at different force levels measured with two myotonometric devices. Physiol Meas. 2011;33(1):65-78.

78. Longo S, Cè E, Rampichini S, Devoto M, Limonta E, Esposito F. Mechanomyogram amplitude correlates with human gastrocnemius medialis muscle and tendon stiffness both before and after acute passive stretching. Exp Physiol. 2014;10(99):1359-69.

79. Miyamoto N, Oda S. Effect of joint angle on mechanomyographic amplitude during unfused and fused tetani in the human biceps brachii muscle. Eur J Appl Physiol. 2005;95(2):221-8.

80. Orizio C, Solomonow M, Diemont B, Gobbo M. Muscle-joint unit transfer function derived from torque and surface mechanomyogram in humans using different stimulation protocols. J Neurosci Methods. 2008;173(1):59-66.

81. Orizio C, Celichowski J, Toscani F, Calabretto C, Bissolotti L, Gobbo M. Extra-torque of human tibialis anterior during electrical stimulation with linearly varying frequency and amplitude trains. J Electromyogr Kinesiol. 2013;23(6):1375-83.

82. Koren K, Šimunič B, Rejc E, Lazzer S, Pišot R. Differences between skeletal muscle contractile parameters estimated from transversal tensiomyographic and longitudinal torque twitch response. Int J Fundam Appl Kinesiol. 2015;47(1):19-26.

83. Ibitoye MO, Hamzaid NA, Wahab A, et al. Estimation of electrically-evoked knee torque from mechanomyography using support vector regression. Sensors. 2016;17(7):1-16.

84. Miramonti AA, Jenkins ND, Oza PD, Weir JP, Cramer JT. Mechanomyographic responses during recruitment curves in the soleus muscle. Muscle Nerve. 2017:56(1):107-16.

85. Uchiyama T, Sakai H. System identification of evoked mechanomyogram from abductor pollicis brevis muscle in isometric contraction. Med Biol Eng Comput. 2013;51(12):1349-55.

86. Dzulkifli MA, Hamzaid NA, Davis GM, Hasnan N. Neural network-based muscle torque estimation using mechanomyography during electrically-evoked knee extension and standing in spinal cord injury. Front Neurorobot. 2018;12:1-11.

87. Shima N, McNeil CJ, Rice CL. Mechanomyographic and electromyographic responses to stimulated and voluntary contractions in the dorsiflexors of young and old men. Muscle Nerve. 2007;35(3):371-8.

88. Trager G, Michaud G, Deschamps S, Hemmerling TM. Comparison of phonomyography, kinemyography and mechanomyography for neuromuscular monitoring. Can J Anesth. 2006;53(2):130-5.

89. Michaud G, Trager G, Deschamps S, Hemmerling TM. Monitoring neuromuscular blockade at the vastus medialis muscle using phonomyography. Can J Anesth. 2005;52(8):795-800.

90. Salminen J, van Gils M, Paloheimo M, Yli-Hankala A. Comparison of train-of-four ratios measured with Datex-Ohmeda's M-NMT MechanoSensor and M-NMT ElectroSensor. J Clin Monit Comput. 2016;30(3):295-300.

91. Watanabe $S$, Nojima I, Agarie Y, et al. Electrically induced mechanomyograms reflect inspiratory muscle strength in young or elderly subjects. Respir Investig. 2016;54(6):436-44.

92. Wessell N, Khalil J, Zavatsky J, Ghacham W, Bartol S. Verification of nerve decompression using mechanomyography. Spine J. 2016;16(6):679-86.

93. Deffieux T, Gennisson JL, Tanter M, Fink M. Assessment of the mechanical properties of the musculoskeletal system using 2-D and 3-D very high frame rate ultrasound. IEEE Trans Ultrason Ferroelectr Freq Control. 2008;55(10):2177-90.

\section{Publisher's Note}

Springer Nature remains neutral with regard to jurisdictional claims in published maps and institutional affiliations. 